\title{
Safe and Efficient Lane Change Maneuver for Obstacle Avoidance Inspired From Human Driving Pattern
}

\author{
Suhyeon Gim ${ }^{\circledR}$, Sukhan Lee ${ }^{\circledR}$, Life Fellow, IEEE, and Lounis Adouane ${ }^{\circledR}$, Member, IEEE
}

\begin{abstract}
One of the most important and fundamental topics in autonomous navigated vehicle research is the lane change maneuver for obstacle avoidance or overtaking maneuver. In the literature, the lane change maneuver path for car-like vehicles has widely been generated with geometrically smooth segments by solving boundary conditions under given constraints. This paper proposes a new method of continuous curvature path generation for the issue of lane change maneuver for obstacle avoidance while solving the clothoids composition problem using an efficient algorithmic procedure. Conventional approaches resorting to mathematical or engineering optimization without considering human-side activity and response may fail to deliver driving performance that is favorable to humans. The novelty of the proposed method lies in its adoption of a human driving pattern, which is non-symmetric and composed of two different modes of avoidance and recovery during the maneuver, and utilizes the property given by an appropriate iterative algorithm which takes into account all the constraints in order to solve the problem. As compared to conventional methods, the proposed method not only provides overall safety for obstacle avoidance, but also exhibits efficiency of increased comfort and human like steering motion during the lane change maneuver. The proposed path planning method is compared to other methods in order to validate its efficiency for safe and smooth obstacle avoidance maneuver.
\end{abstract}

Index Terms-Continuous curvature path, human driving pattern, clothoid, lane change maneuver, obstacle avoidance, passenger comfort.

This work was supported in part by the Robocarechair: A Smart Transformable Robot for Personal Care of the Korea Institute for Advanced Technol-ogy (KIAT) sponsored by MOTIE under Grant KIAT P0006886, in part by the AI Graduate School Program under Grant 2019-0-00421, in part by the ICT Creative Consilience program of the Institute of Information and Communication Technology Planning and Evaluation (IITP) sponsored by MSIT under Grant IITP-2020-0-01821, and in part by the French research program Investissements $d^{\prime}$ Avenir through the IMobS3 Laboratory of Excel-lence under Grant ANR-10-LABX-16-01. The Associate Editor for this article was A. Amditis. (Corresponding author: Sukhan Lee.)

Suhyeon Gim was with the Department of Electrical and Computer Engineering, SungKyunKwan University, Suwon 16419, South Korea, and also with the Institut Pascal, University Clermont Auvergne, 63000 ClermontFerrand, France. He is now with the Hyundai Heavy Industry Group, Seoul 03058, South Korea.

Sukhan Lee is with the Departments of Artificial Intelligence and Electrical and Computer Engineering, SungKyunKwan University, Suwon 16419, South Korea (e-mail: 1sh1@skku.edu).

Lounis Adouane is with the Heudiasyc UMR CNRS/UTC 7253, 60200 Compiegne, France, and also with the Institut Pascal, UMR UCA/ SIGMA 6602, 63000 Clermont-Ferrand, France (e-mail: lounis.adouane@ utc.fr).

\section{INTRODUCTION}

$\mathbf{T}$ HE autonomous navigated vehicle and driver assistant system has been of interest to robotics researchers and vehicles engineers for several decades [1]. A substantial amount of research has been conducted on path generation for safe maneuvering or obstacle avoidance in the broad field of autonomous ground vehicle (AGV) and advanced driver assistance systems (ADAS). For a vehicle to perform a safe motion during various risky situations, one of the most fundamental path/trajectory maneuvers to be performed is the lane change for obstacle avoidance. In order to avoid any collision with approaching obstacles on a road environment, the vehicle must generate a new path and then follow that path by controlling its steering and speed [2]-[4]. Thus, an important requirement for the generated path is that it should be sufficiently precise and easy to be followed by a vehicle with nonholonomic motion constraints. Many research groups have attempted path generation using interpolation or composition of geometrically smooth segments [5]-[9]. Meanwhile, even if the path is generated by some optimal or sub-optimal path generation scheme (e.g., the shortest length or minimum spent time or fuel consumption), the generated path can lead to discomfort for the passengers when the vehicle's motion involves high acceleration or jerking [10]-[12]. Hence, to follow a smooth path for a car-like vehicle, the continuous curvature path (CCP) has been investigated by many researchers since it has smooth steering control while providing comfort to passengers [7], [10], [13]. The CCP is geometrically smooth not only in the Cartesian space, but also in its curvature over the entire length. Thus, in contrast to the geometrically smooth path, the $\mathbf{C C P}$ has a distinct advantage in that the obtained vehicle's path curvature is directly controlled via the vehicle's front wheel angle. Thus, its steering control along the path is assured to be smooth [14]. The obtained path is particularly useful for a nonholonomic car-like vehicle to follow without having to stop to reorient its front wheels.

Regarding works related to $\mathbf{C C P}$, efficient path generation methodologies have been developed over the past few decades, as follows. First, Dubins [15] (Dubins path) and, Reeds-Shepp [16] (RS path) proposed smooth path models for nonholonomic vehicles yielding the shortest travel length; however, they both lacked curvature continuity. Fraichard-Scheuer [17] (FS path) presented a path model comprised of lines, circular arcs, and clothoids, resulting in a 
path that has continuous curvature with a fixed upper-bounded curvature; as well as upper-bounded sharpness in the absence of obstacles.

However, the difficulty in dealing with the clothoid-based path generation problem is that it is very hard to obtain a closed form that is analytically explicit. Thus, it requires a large number of numerically complex iterations to converge to a result. In order to cope with the complexity in the algorithmic procedure and to enable the generated paths to have quality and comfort favorable to humans, some works have aimed for enhanced path generation methods by investigating human driving patterns. For example, in [18] and [19], the desired paths were made by cubic splines in lane change maneuvers. In both works, the human steering pattern was divided into avoidance and stabilization, and each steering control rule was then applied by minimizing the lateral deviated distance of the vehicle to the desired path. In [20], the steering control was executed by tuning a few gain parameters and comparing the resultant steering control with those of human driver models. The above studies focused on the design of the steering control from human driving data. In [21], a human driver model for the steering control to follow a curved lane was formulated so as to enhance the fidelity. The results indicated that the human driver used preview information ahead of the recognized curvature on the road and controlled the steering of the vehicle while taking reaction time delay into account. In [22], an adaptive steering controller was applied to an autonomous lane change maneuver in order to avoid a static obstacle, where the desired trajectory was generated by a cycloid. When the desired trajectory was made to avoid the static obstacle, a circular path was considered with a maximum steering angle such that the path would always keep a minimum distance from the obstacle.

Some studies have also been performed to implement human driver intentions or moods into a steering control strategy. For example, biomimetic motion planning was performed in vehicle speed control [23]. Human driving patterns were addressed in [24] and a vehicle steering control strategy was discussed in [25]. In [24], the controller adjusted the steering control gain according to the driver mood from aggressive to leisurely. The authors in [25] made a steering controller by differentiating the degree of emergency in the lateral collision avoidance control, from emergent steering for avoiding collisions to gentle steering to stabilizing for an approaching lane. The proposed schemes were tested on a severe lane change track of ISO 3882-2. The authors in [26], [27] extracted control parameters from experimental investigations in which an expert human driver combined longitudinal and lateral controls using jerk information during cornering maneuvers. In [28], steering and braking control was implemented for collision avoidance, where the reference path was generated by a combination of line and arc segments. In the methodology used, the reference path was non-continuous, so a controlled result could not actually be obtained and the collision safety could not be ensured either. The authors in [29] investigated drivers' patterns in heavy vehicle's steering and found that experienced drivers perform cornering motions with more safety margin and low lateral acceleration.
In [30], a CCP was investigated with human factors. It presented a smooth and easily drivable path (the Wilde path, in reference to the name of the author) composed by four identical clothoids using rational approximation functions. It also imposed a minimum sharpness (or rate of curvature) constraint so as to closely resemble natural and safe human driving. The algorithmic procedure was fast, but the path was only useful for lane change maneuvers in obstacle-free environments. In the lane change maneuvering problem, two identical $C$-shaped curves are composed to make a $S$-shaped curve under the constraint such as the golden section rule [8], the minimum sharpness [30], or vehicle's dynamics stability [31], where a $C$-shaped curve with a continuous curvature as well as geometry and orientation continuity, can be composed by two clothoids (cf. Fig. 6). Related to the clothoids composition in the path generation problem [32], [33], a parametric CCP algorithmic solution was proposed involving iterative regulation of the clothoid parameters. The work has a weakness in its algorithmic efficiency, i.e., the number of iterations increased in order to obtain a solution according to the given boundary conditions. Even if the proposed solutions in [30] or [32] assure smooth paths for nonholonomic vehicle motion, they are not optimized for human (passenger) comfort. Furthermore, it is better to consider the influence of environmental conditions on human comfort and steering patterns from urgent, imminent responses (quick/sharp turning) to relaxed, quiet responses (slow/gentle turning).

\section{Major Purpose and Motivation}

In this paper, we consider a lane change maneuver for static obstacle avoidance in a straight structured road. The structured road includes a couple of lanes for each vehicle to follow and it is considered that the obstacle's size/shape does not exceed the road width. In such a configuration, the purpose of our work is to generate a safe and efficient trajectory for car-like vehicles and ensure that the obtained path/trajectory possesses mathematically provable properties and low computational features while guaranteeing the comfort of the passengers. It is difficult for the path generation scheme to satisfy the requirements of both engineering optimization and human preference. For example, the fastest or shortest (least fuel consumption) often leads to discomfort for human passengers [9], [11], [12]. In addition, for lane change maneuver, the issue of when to start turning the steering wheel and where to pass near the obstacle are not easily solved from only the computational performance measure, since such vehicle motion could increase fear and affect human passengers.

In order to reach a good compromise between the perspectives of both engineering and human sides, this paper attempts to find a new path generation scheme by adopting human driving pattern while using the good expertise of human drivers. The authors' previous works [32]-[34] deal with CCP for nonholonomic car-like vehicles, and cover various geometric boundary conditions by composing clothoid segments with parametric variation procedure and the corresponding geometric convergence criteria. The issue of lane change for static obstacle avoidance could be tackled under the framework 
of the $\mathbf{C C P}$ problem; however, if considering practical application on the road, the problem requires additional conditions or constraints as follows. The point to include at first is where (or when) to start turning to avoid and where (when) to rendez-vous with the other side lane, i.e., how to define the full boundary conditions for the $\mathbf{C C P}$ problem in the maneuver. The second point is how and what to choose as the path from numerous solution candidates satisfying the boundary conditions at the start and final poses, i.e., how/what to adopt the optimization criteria in the algorithmic procedure. Another point to be specified is how much the parameters should be allowed and bounded while satisfying the given performance measure. In order to identify a solution for those practical points, this work designs several experimental configurations under control, then gathers human driving data and aims to extract significant patterns and features.

The proposed algorithm finds a human-inspired $\mathbf{C C P}$ (h-CCP) solution of multiple clothoids. The solution is obtained from parametric iterative procedure under performance optimization. The optimization rule is guided by human driving patterns which were tested and obtained from experienced human drivers.

This paper is organized as follows. In the next section, the CCP generation scheme is introduced and experimental investigations on human driving patterns are presented. In section III, the problem statement and solution for the lane change maneuver with obstacle avoidance are addressed with algorithmic descriptions. In section IV, the proposed path solution is compared with other methods and the work is then concluded with a discussion and final remarks.

\section{EXPERIMENTAL INVESTIGATION OF HUMAN DRIVING PATTERNS}

\section{A. Continuous Curvature Path Model}

A basic path generation problem is defined by two boundary conditions, with one being the initial pose and the other being the final pose. The path solution connects two boundary poses and simultaneously satisfies the orientation and curvature conditions.

A property of a clothoid is that its curvature is continuous, which either increases or decreases along the path. Various types of formulations are possible for the curvature rate (or sharpness) such as polynomials; or exponential or trigonometric function. However, the $1^{s t}$ order form, relying on a constant sharpness, is well known for not only its simplicity of computation but also its phenomenal similarity to a real vehicle actuation system. An elementary clothoid is defined by the parametric forms given by [32],

$$
\begin{aligned}
& \kappa(s)=\alpha s, \\
& \theta(s)=\int_{0}^{s} \kappa(u) d u, \\
& x(s)=\int_{0}^{s} \cos \theta(u) d u, \\
& y(s)=\int_{0}^{s} \sin \theta(u) d u,
\end{aligned}
$$

where, $s$ is the curvilinear distance traveled along the path, $\alpha$ is the sharpness (or rate of curvature) for curvature $\kappa(s)$.
Eq. (1) shows that the curvature increases or decreases linearly according to a constant sharpness $\alpha$ and the orientation $\theta$ in (2) changes with the integration of the curvature given in (1).

When a clothoid is defined, the relations between the parameters are presented as follows,

$$
\kappa=\sqrt{2 \delta \alpha}\left(\text { or } \delta=\frac{\kappa^{2}}{2 \alpha}\right), \quad s=\sqrt{\frac{2 \delta}{\alpha}} .
$$

where the parameters $\alpha, \delta, \kappa$ and $s$ are the values at the end point of the clothoid and $\delta$ is defined as the amount of orientation change through the whole length $\left(\right.$ deflection $\left.^{1}\right)$ [10], [30].

Note that, as shown in Eq.(5), even though there are three parameters, there are only two degrees of freedom. When a clothoid can be adjusted by one parameter among $\alpha, \kappa$ and $\delta$, with another parameter fixed, then the last third parameter is determined from the other two parameters. This parametric adjustment facilitates the resolution of the $\mathbf{C C P}$ problem in an iterative manner as described in previous works by the authors [32], [34]. However, the procedure of parametric adjustment requires a number of iterations to converge to a solution; therefore, it is better to give an additional constraint so as to increase the calculation speed. Another issue for the procedure is that the resulting solution does not consider the human factor. In this regard, integrating human driving characteristics in the algorithmic procedure, could be beneficial for obtaining a solution similar to that achieved by a skilled human driver. This work aims to propose a solution methodology for CCP generation for lane change maneuver. An important performance objective is to ensure that the algorithmic solution includes natural and human favorable driving patterns. In this respect, an experimental investigation on human driving patterns provides guidance or constraints to obtain the desired solution.

\section{B. Experiments for Acquiring Human Driving Pattern}

In order to find human driving patterns, three experiments were designed and set up. The first set of experiments were planned to acquire obstacle avoidance pattern for small obstacles in open parking. The second set of experiments aimed to examine lane change maneuver for large obstacle avoidance on a structured road, while the third set of experiments were performed to gather steering motions for various turning conditions in an obstacle-free environment.

Note that the results of these experiments were not meant to be generalized to all humans, but only aimed to find a prominent or noticeable patterns for the sampled human applicants. Thus, for this purpose and before starting any test, each human driver was instructed on how to perform the test. All of the guidelines and constraints from each experiment were designed to determine useful human driving patterns which were then implemented into the h-CCP solution (cf. section III).

The experimental vehicle is shown in Fig. 1. The dimension of the experimental vehicle is $1.5 \times 1.4 \times 3.1$ (width $\times$ height $\times$ length in $[\mathrm{m}]$ ) with a wheelbase of $2.18 \mathrm{~m}$. It has sensors for

\footnotetext{
${ }^{1}$ Thus, deflection is positively increased by $s$ accumulation.
} 


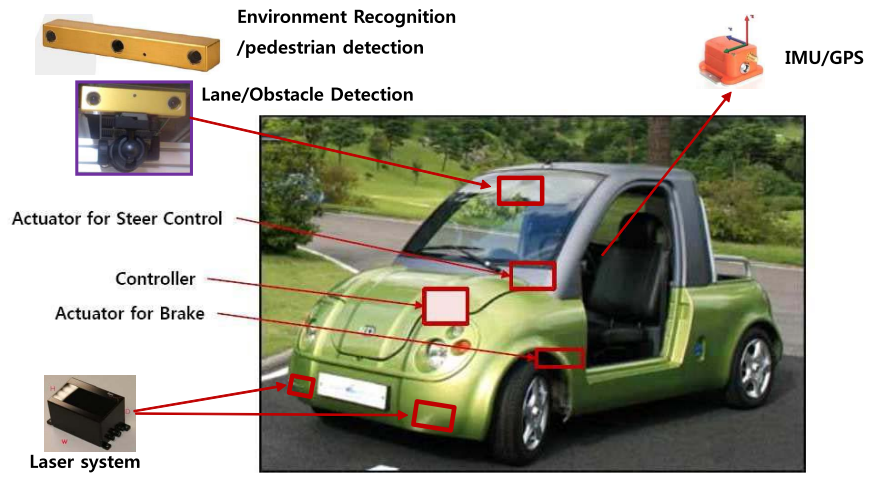

Fig. 1. Experimental vehicle.

recording the steering/wheel angular, position and orientation by integrating data from the IMU/GPS and odometers.

1) Experiment 1: Obstacle Avoidance Maneuvering by Human Driver: An obstacle avoidance test was performed by human drivers. The obstacle is placed on the front side of the vehicle at the initial position, and the driver is then told to avoid the obstacle on the left-side and then return to the center line until reaching the target position. The obstacle is small with dimensions of $0.5 \times 0.5 \times 1.0($ width $\times$ length $\times$ height $)$ in $[\mathrm{m}]$ so that a human could recognize the size and shape of the entire object as well as its position.

First, twenty persons were applied to this experiment and each driver performed at least 3 trials. Among them, five drivers were chosen for the next experiments because they showed more than 2 coherent dataset. ${ }^{2}$ The age range of applicants' is from 22 to 45 years, and the pure driving experience is from six months to 10 years. All drivers were instructed to speed up to $30[\mathrm{~km} / \mathrm{h}]$ from the start and drive the vehicle's travel length as short as possible without substantial discomfort to the driver. Each driver attempted the test several times, then chose the most satisfying result according to his or her judgement. ${ }^{3}$

In Fig. 2(a) and (b), the geometric setup for the given Exp.1 with its picture shot is shown, where the initial position of the vehicle is its origin in the coordinate frame; the first obstacle (Obst.1) is located $17 \mathrm{~m}$ from the front of the vehicle, while the other obstacle (Obst.2) is located on the left side of the target so as to guide the driver to drive the distance to the target position of $32 \mathrm{~m}$ behind the Obst.1. In Fig. 2(b), four geometrical parameters are defined for evaluating the performance of the obtained path data for each human driver, where the avoidance distance $\left(\boldsymbol{d}_{\boldsymbol{a v d}}\right)$ refers to the distance between the obstacle and the position at which the vehicle starts to turn [35], and the return distance $\left(\boldsymbol{d}_{\boldsymbol{r t} n}\right)$ is the distance between the obstacle and the return position which is the same as the initial vehicle direction. The minimum distance, $\boldsymbol{d}_{\min }$ is found at the position where the vehicle's right side is the

\footnotetext{
${ }^{2}$ There can be various categorizations of driver samples. In [12], drivers were classified into three different categories as Slow, Medium and Aggressive drivers.

${ }^{3}$ Every human driver shows better/efficient pattern as his/her knowledge and experience on the driving route are accumulated. The best of the most samples for each candidate was chosen not by author, but by driver.
}
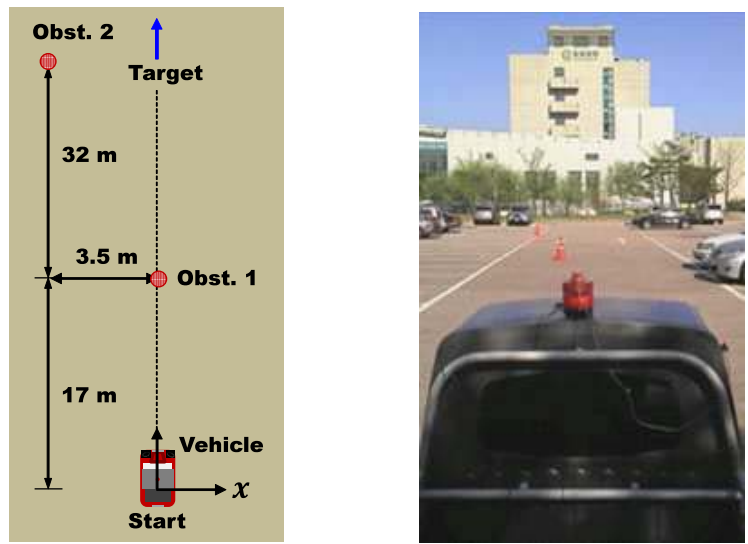

(a) Experimental setup and its scene

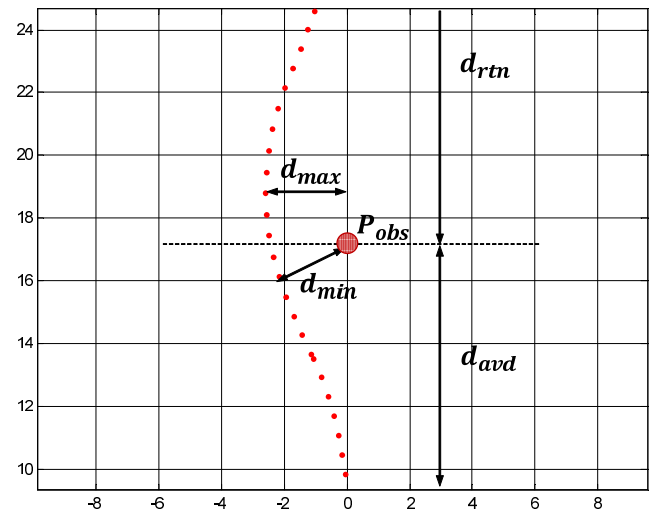

(b) Path evaluation parameters

Fig. 2. Obstacle avoidance by human drivers: Exp.1.

TABLE I

Results ANalysis of Exp.1 ( $[\mathrm{m}])$

\begin{tabular}{|c|l|l|l|l|l|l|}
\hline Appl. & $\begin{array}{l}(1) \\
\boldsymbol{d}_{\boldsymbol{a v d}}\end{array}$ & $\begin{array}{l}(2) \\
\boldsymbol{d}_{\min }\end{array}$ & $\begin{array}{l}(3) \\
\boldsymbol{d}_{\max }\end{array}$ & $\begin{array}{l}(4) \\
\boldsymbol{d}_{\boldsymbol{r t n} \boldsymbol{n}}\end{array}$ & Total $^{\star}$ & $\begin{array}{l}\text { Period } \\
\text { (years) }\end{array}$ \\
\hline (a) & 6.8 & 1.2 & 1.3 & 5.8 & 15.1 & 10 \\
\hline (b) & 7.8 & 1.8 & 1.9 & 7.1 & 18.6 & 3 \\
\hline () & 8.0 & 1.7 & 1.8 & 7.9 & 19.4 & 5 \\
\hline (d) & 10.3 & 2.1 & 2.4 & 9.0 & 23.8 & 1.5 \\
\hline () & 12.8 & 3.7 & 3.8 & 12.2 & 32.5 & 0.5 \\
\hline
\end{tabular}

closest to the obstacle and the lateral distance $\boldsymbol{d}_{\max }$ is the maximum lateral position from the line of $x=0$ in the local coordinate frame.

In Exp.1, From the Start position, the human driver begins to speed up until turning. In order to avoid collisions, the driver rotates the steering handle to the left, then return to its initial direction so as to approach the given target. When turning to avoid as well as returning, each driver tries to his or her best not to amplify discomfort.

Table I records the obtained path data by the four parameters defined in Fig. 2(b). Its evaluation is performed by summing all the parameter values for each human.

In Table I, five applicants are listed up by evaluated points, Total. As the point of Total is low, the driving performance 
is high. Thus, drivers are ranked from applicants (a) to (e) for Exp.1. Even if all of the parameters differ between all applicants, a pattern could be found that the most experienced driver (a) records the lowest point of Total, and thus the highest performance, whereas the least experienced driver (e) records the worst performance from the point Total.

From Table I, it is also evident that experienced drivers (with more than three years driving experience) follow paths with shorter distances to the obstacle and generate a more inside turning path than less-experienced drivers. The four parameters are closely related to the total length of the path, since as all parameter values increase, the total length for maneuvering also increases. From the obtained evaluation result, a human driving pattern could be derived such that a more experienced driver makes a shorter obstacle avoidance path by having closer turning around the obstacle than less experienced drivers. The tests in Exp.1 report the shape of the path for expert human driving, so they requires the consideration of human driving patterns according to vehicle speed.

2) Experiment 2: Lane Change for Obstacle Avoidance: In experiment 2 (Exp.2), lanes on city roads are used for the lane change maneuvering test and an obstacle (parked vehicle) was placed on the right side of the road $42 \mathrm{~m}$ away from the front of the vehicle start position. The obstacle is large enough that a human driver could not see beyond the obstacle (i.e., the obstacle blocks the driver's view), where one could not recognize the whole shape of the obstacle, and where it was also difficult to expect what other obstacles may be behind the obstacle. The drivers include five drivers, who were selected from Exp.1.

In this experiment, each driver was instructed to increase their vehicle speed as much as possible and then maintain that speed (the avoidance speed $\boldsymbol{v}_{\text {avd }}$ ) until the vehicle began to turn at the steering angle. The driver was also told to approach the obstacle as close as possible and then keep going without pushing the accelerator or brake pedal during the lane change maneuver. These constraints are used to investigate the desirable relative distance between the vehicle and the obstacle as well as to analyze the geometric pattern of the resultant path. Each driver is also instructed to test the same lane change maneuvering with five steps of $\boldsymbol{v}_{\text {avd }}$ as slowest, slow, middle, fast, and fastest. Although each step for the $\boldsymbol{v}_{\boldsymbol{a v d}}$ is determined by each human driver, and is thus different from those of the other drivers, the pattern between $\boldsymbol{v}_{\boldsymbol{a} \boldsymbol{v} \boldsymbol{d}}$ and $\boldsymbol{d}_{\boldsymbol{a} \boldsymbol{v} \boldsymbol{d}}$ could be derived.

With the experimental setup depicted in Fig. 3, the results for human lane change maneuvering are shown in Fig. 4 for the path (a), speed (b), steering (c), and captured scenes from the driver side (d). All of the drivers began to turn from seven seconds and maintained the maneuvering for eight to 10 seconds. From the driver's view (cf. Fig. 4(d)), the sight behind the obstacle could be recognized ( $5^{\text {th }}$ scene) a few seconds after the beginning of the avoidance, and this seems to make the whole path non-symmetric along the travel time or distance. A steering motion pattern could be found (especially for more experienced drivers, Driver (a) to (C) in Fig. 4(c), where two peaks are prominent through the maneuver. The preceding

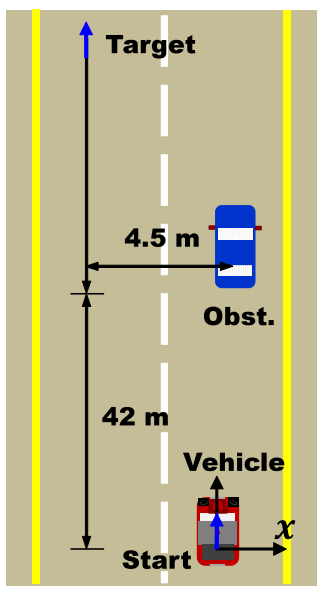

(a) Experimental setup

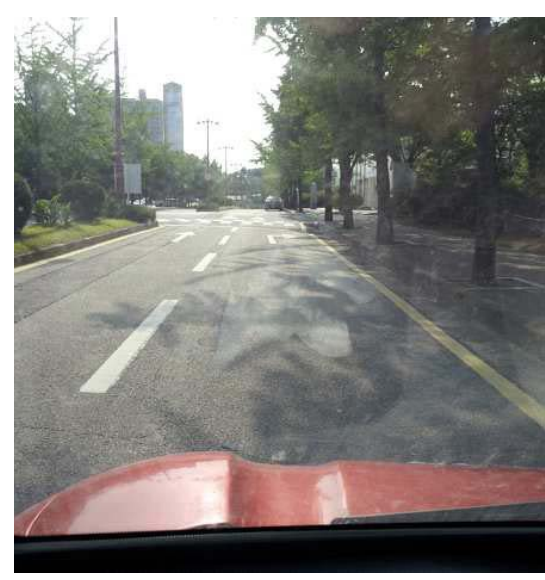

(b) Driver's view
Fig. 3. Lane change for obstacle avoidance: Exp.2.

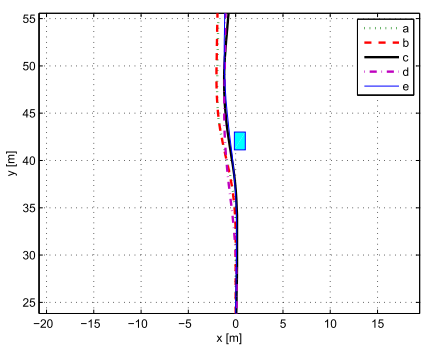

(a) Paths for five drivers (b) Speeds for five drivers
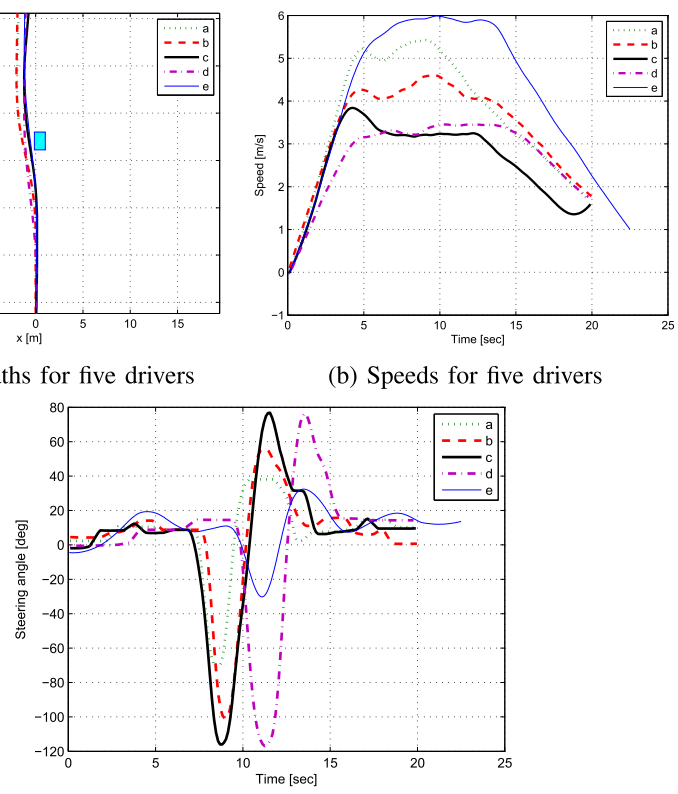

(c) Steering angles for five drivers

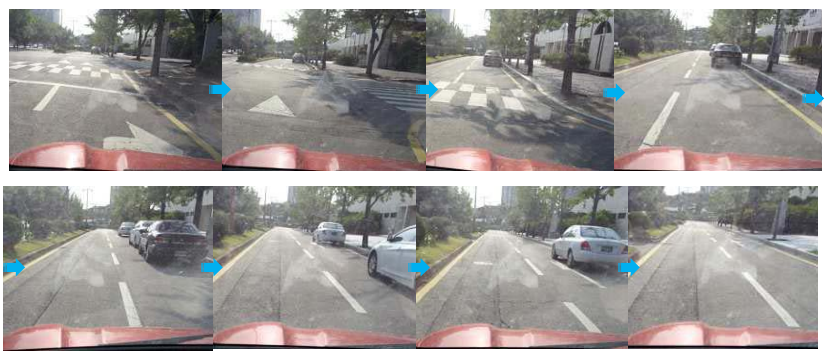

(d) View of driver

Fig. 4. Human driving data results for lane change maneuvering.

peak is sharper and higher than the following peak. It is remarkable that the lane change maneuver of a human driver could be non-symmetric during the entire steering motion, which is not reflected in the previous approaches where a symmetry has been used as in [8], [20], [28] as well as in [30]. It is valuable to adopt this important feature into the algorithmic procedure in order to compose the proposed path. We will 


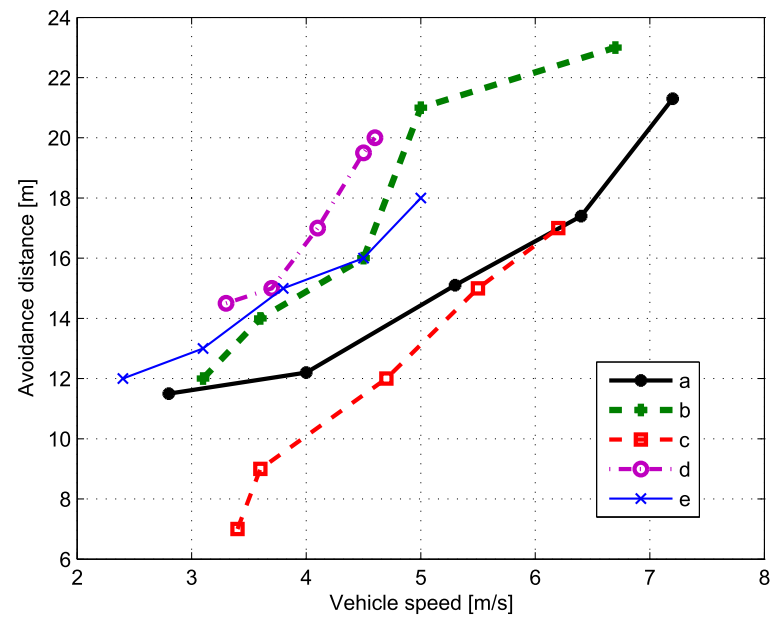

Fig. 5. Avoidance distances according to vehicle speeds for the five drivers.
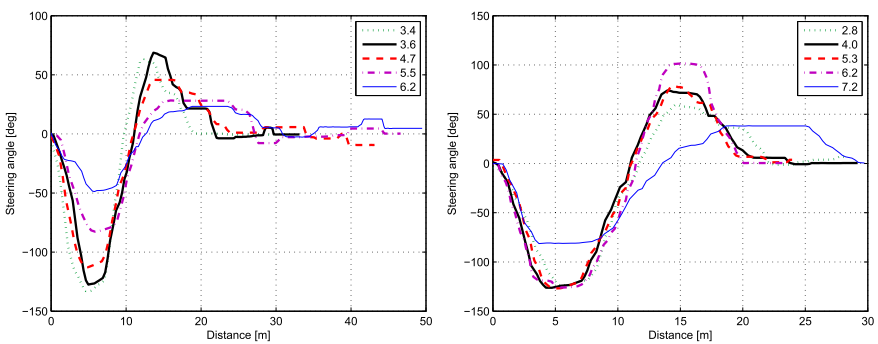

Driver (a)

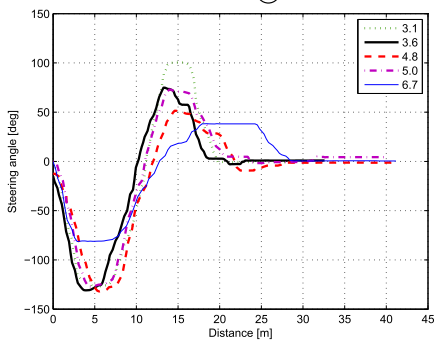

Driver (b)

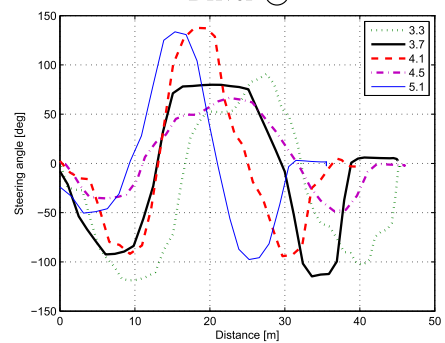

Driver-(c)

Driver (d)

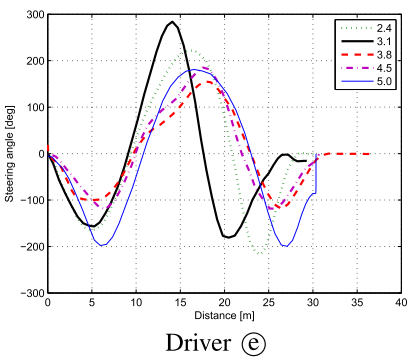

Fig. 6. Steering patterns for drivers with different speeds $[\mathrm{m} / \mathrm{s}]$.

name these two different steering motions as avoidance and recovery modes.

Fig. 5 presents the five results of the steering angle along the travel length for the five drivers, where each subfigure itself has five datasets of steering angle record for five different $\boldsymbol{v}_{\boldsymbol{a v d}}[\mathrm{m} / \mathrm{s}]$. In Fig. 5, the avoidance distance $\boldsymbol{d}_{\boldsymbol{a v} \boldsymbol{d}}$ according to the vehicle's maximum speed $\boldsymbol{v}_{\boldsymbol{a v d}}$, increases for all of the drivers from (a) to (e). The steering records of each driver (a) to (e) are respectively displayed in five subfigures of Fig. 6, and each subfigure contains several test results indexed by different $\boldsymbol{v}_{\boldsymbol{a v d}}$ values. In order to effectively extract the steering pattern, only the steering data during turning motion are plotted after being aligned on the same origin position of $x$-axis. From the above records, it is found that human drivers tend to begin turning their steering wheel earlier as the vehicle speed increases. This pattern can be easily deduced from the fact that human driver feels more danger of the risk of collision as the obstacle approaches closer and comes faster. Thus, the proposed path should take into account this risk of human feeling, i.e., the speed of the relative distance between the vehicle and the obstacle.

Choosing the data set of driver-a shown in Fig. 5 (black lines with dot markers), the relation between $\boldsymbol{v}_{\boldsymbol{a v d}}([\mathrm{m} / \mathrm{s}])$ and $\boldsymbol{d}_{\boldsymbol{a v d}}([\mathrm{m}])$ can be modelled by line fitting as follows,

$$
\boldsymbol{d}_{\text {avd }}=2.67 \boldsymbol{v}_{\text {avd }}+1.31 \text {. }
$$

Note that even if the pattern shown in Eq.(6) is not exactly the same as those of other drivers, it could be generalized that $\boldsymbol{d}_{\boldsymbol{a v d}}$ increases proportionally to $\boldsymbol{v}_{\boldsymbol{a v d}}$ for all human drivers. This function of pattern was formulated from only a small number of drivers, but this data could be useful for providing guidance regarding the proposed path generation algorithm.

3) Experiment 3: Driving on an Obstacle-Free Road: For the third experiment, free road driving was executed in the SungKyunKwan University campus of Korea. The road track has one lane per path and all experimental data are gathered under controlled obstacle free conditions. Even though constraints were given for Exp.1 and Exp.2, no constraint was set for Exp.3 for the driving and the drive-recorded path, steering, and velocity of the vehicle prior to stopping.

This experiment was designed to understand human driving patterns for various cornering or lane change conditions in a structured road (cf. Fig. 7). Three experienced drivers (a), (b) and (C) were selected, and each driver performed a pretest on the given three tracks several times so as to become accustomed to the complete road geometry and ground condition of each track. Each driver was instructed to drive the vehicle with medium time, not agressive or slow way [12] while preserving comfort. Three tracks, track \#1, \#2, and \#3, were tested in order to collect free driving data, where track \#1 includes straight double lanes, track \#2 has a cornering road geometry of one steep and one less steep curve, and track \#3 has a squared shape, thus many $90^{\circ}$ crossings were involved in the road geometry. One representative record for each track $^{4}$ was chosen for analysis as follows.

Fig. 8 presents the records obtained for steering angle and lateral acceleration during the driving on tracks, as shown in Fig. 7. In each path, left lane change, right lane change, left cornering and right cornering are referred to as $\ell L C, r L C$, or $\ell C o, r C o$ respectively. The three tracks (Track \#1 to \#3) differ from each other in terms of geometrical complexity and cornering angles; however, human drivers showed a common feature in the obtained dataset as follows. At first, as the cornering angle is large, the dynamic range of the steering

\footnotetext{
${ }^{4}$ It is not found any predominant discriminated pattern in the data obtained by three drivers, thus the results by different drivers are shown in the following figures.
} 


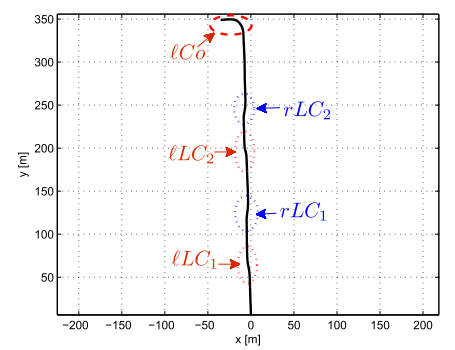

(a) Track \#1 by driver-(a)

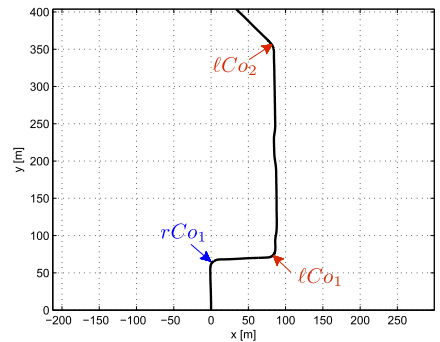

(b) Track \#2 by driver-(b)

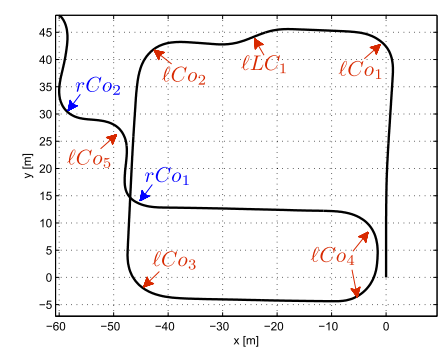

(c) Track \#3 by driver-(C)

Fig. 7. Path results for Exp.3 tracks.

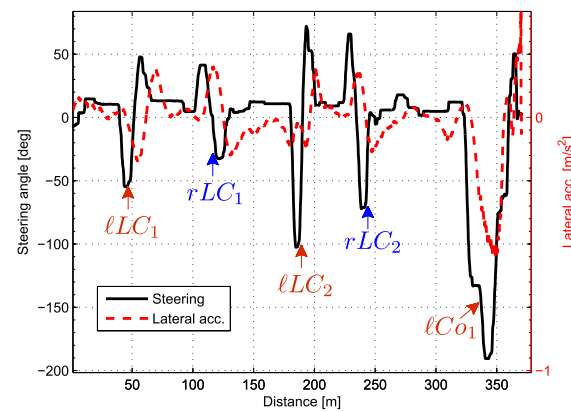

(a) Track \#1

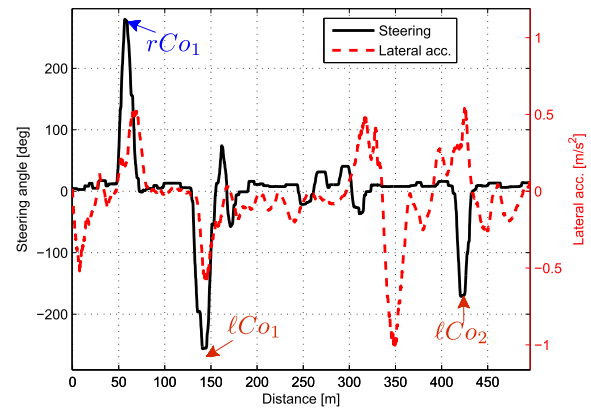

(b) Track \#2

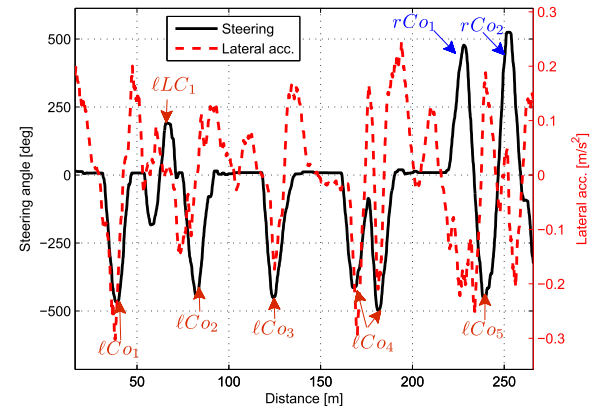

(c) Track \#3

Fig. 8. Steering and lateral acceleration results for Exp.3.

angle also increases. Secondly, as the steering angle changes rapidly, the lateral acceleration grows rapidly as well. Thirdly, as the amount of steering is high (or low), the amount of lateral acceleration has a high (or low) value. ${ }^{5}$ Since the lateral acceleration serves as a major indicator for the passenger's ride comfort, it is manifest that the steering motion needs to be slow and have a low peak value (low maximum or high minimum) if the vehicle maintains its speed, c.f. $a_{\text {lat }}=\kappa V^{2}$ ( $a_{\text {lat }}$ : lateral acceleration, $\kappa$ : curvature and $V$ : speed). These analyses appear to have a few discrepancies in the dataset. The possible causes are from the spring/damper effects of the mechanical linkage and also tyres lead to delays for the response of the vehicle. Another factor to the distorted value of the lateral acceleration is the irregularities on the road surface such as manhole structures and small craters, which cases large shock to the vehicle (especially in the latter part of Tracks \#2 and \#3).

4) Observed Patterns on the Obtained Results: From the analysis results of Exp.1 to 3, the following properties are summarized in terms of lane change maneuver for obstacle avoidance.

Property 1: Human driving patterns during lane changing for obstacle avoidance

i. Efficient path achieves a shorter travel length with closer turning around the obstacle.

ii. Avoidance distance increases as the vehicle speed increases.

iii. Steering rate $(\dot{\gamma})$ is proportional $(\alpha)$ to the relative velocity of the vehicle to the obstacle, i.e., $\alpha \propto v_{\text {rel }}$ (or $j \propto \alpha$ ).

iv. Steering rate changes between before and after avoidance, so it needs to be dealt with two different modes.

v. Steering motion of the after-avoidance has a lower and wider shape than the before-avoidance, where this pattern comes from the relaxed mood of the human [24].

From these findings in Property 1, the lane change path for the obstacle can be divided into two problems of avoidance and recovery mode paths by different optimization strategies.

In particular, the steering patterns of avoidance and recovery modes differ in that the steering of avoidance mode has sharper shape and shorter travel length than the recovery mode (cf. Fig. 6) and they are non-symmetric (cf. Section III). This paper aims at connecting a path to h-CCP problem and then regenerating or mimicking the path as driven by a human expert. In order to provide optimization strategies for the $\mathbf{h}$ CCP problem, Property 1 is utilized to formulate the solution constraint in the algorithmic procedure.

From the analysis of human driving patterns, it can also be found that a curvature in the path is closely related to the human motion of steering (cf. Exp.3). In order to formulate the problem of $\mathbf{h}-\mathbf{C C P}$ with performance objective to be optimized, it needs to summarize the relations between clothoid parameters, steering angle and human motion, and these relations are listed with corresponding performance measures in Table II.

In Table II, each item in the path is expressed by $\alpha, \gamma$ or performance measures, where $\wp_{r}, \wp_{s}$ and $\wp_{t}$ imply the

\footnotetext{
${ }^{5}$ These findings are important in the respect that the load geometry, human steering, its resultant acceleration and human feeling of comfort are linked each other. Thus the steering pattern (or path generation method) should consider the load geometry (or obstacle avoidance maneuver) for the better comfort.
} 
TABLE II

Relation Between Path, SteEring AND Human Behavior

\begin{tabular}{ll|ll|ll|ll}
\hline \multicolumn{2}{c|}{ path } & \multicolumn{2}{c|}{ steering } & \multicolumn{2}{c|}{ human } & \multicolumn{2}{c}{ p. measure } \\
\hline curvature & $\int \alpha d s$ & angle & $\gamma$ & work & $\int \tau d \theta$ & rotation & $\wp_{r}$ \\
sharpness & $\alpha$ & ang. vel. & $\dot{\gamma}$ & power & $\tau d \theta$ & speed & $\wp_{s}$ \\
torsion & $\frac{d \alpha}{d s}$ & ang. acc. & $\ddot{\gamma}$ & torque & $\tau$ & torque & $\wp_{t}$ \\
\hline
\end{tabular}

amounts of rotation, speed and torque for steering respectively. Note that the torsion (rate of sharpness) corresponds to the angular acceleration of the steering or the steering torque exerted by human arms. Thus, the torsion of the given path affects the amount of the steering torque. With the given notations, a steering work for human $\mathcal{W}_{s t}$ is defined as follows.

$$
\mathcal{W}_{s t}=\int_{t_{1}}^{t_{2}} \tau \gamma d t=\int_{s_{1}}^{s_{2}}|\tau \alpha| d s,
$$

where $\tau$ is the torque exerted by human arms and $\mathcal{W}_{s t}$ can be obtained by torque integration between two states between times $t_{1}$ and $t_{2}$, or $s_{1}$ and $s_{2}$. Eq.(7) implies that the steering work is determined by the sharpness variation and steering rotation. In order to reduce steering work, sharpness variation or steering rotation should be minimized. In the formulation and solution derivation of the problem, the above terms and properties are used in the section III.

\section{Problem Definition and Solution Derivation}

In this section, the h-CCP problem and its clothoid based iterative solution is integrated to realize human driving patterns. The human driving pattern enables the formulation of the h-CCP problem and its solution by providing guidance and constraint while accounting for the passengers' ride comfort.

Different than the conventional problem, this problem does not require all of the information about the initial and final poses, where only the obstacle position and lane width are given. This is a practical consideration for lane change task in normal straight road based on the fact that it is only concerned with aligning the vehicle to the lane direction before/after avoidance for any driver. Thus, in this problem, the distance between the vehicle and the obstacle before avoidance i.e., $\boldsymbol{d}_{\boldsymbol{a v} \boldsymbol{d}}$ (Eq.(6), and the final position after avoidance needs to be additionally obtained under the given constraint of lateral road width. Furthermore, the obstacle and vehicle are modeled by a circle [36], [37] in which each circle encompasses the obstacle's geometric shape and vehicle respectively. Using the circular model, the safe path with collision avoidance can be specified on the equivalent obstacle boundary which contains the obstacle and vehicle circles. From the conditions and restraints described above, the $\mathbf{h}-\mathbf{C C P}$ problem is defined as follows.

Problem Definition: h-CCP Generation for Lane Change Maneuvering: In a straight lane road, when a static obstacle is detected in front of the vehicle, a lane change path should be generated with minimum lateral acceleration ${ }^{6}$ (that is maximum comfort), where only the lateral distance and

\footnotetext{
${ }^{6} \mathrm{~A}$ lateral acceleration felt by the passenger in the vehicle is defined by $a=\kappa v^{2}$.
}

obstacle boundary are given as geometric constraints. Here, it is assumed that the obstacle size does not exceed the lane width and the obstacle is sufficiently far from the vehicle to be avoided. Under the geometric constraints described above, a feasible CCP with the minimal number of clothoids should be found while satisfying the given boundary configurations. The vehicle speed is assumed to be constant in the obstacle avoidance as performed in Exp.1 and 2.

In order to solve the h-CCP problem, it needs to be divided into two subsequent problems, Problem A and Problem B, where each sub-problem is for the avoidance and recovery modes, respectively. Here, the terminologies, avoidance and recovery are named from the driver's intention to vehicle motion, where avoidance refers to the vehicle avoiding an obstacle and recovery refers to a vehicle's orientation (heading) recovering to its initial orientation (heading). The two sub-problems share a common boundary condition (meeting pose $\left.\boldsymbol{p}_{\boldsymbol{m}}\right)$ at the transition between avoidance and recovery mode, where the position, orientation, and curvature in the two paths are all the same.

\section{A. Problem A}

This subsection deals with the avoidance mode (Problem A). It is assumed that an obstacle is stationary and located in front of the vehicle such that if the vehicle does not change its lane, a collision will be unavoidable. As described in Exp.2, the avoidance situation is urgent as the driver/passenger is threatened. The highest priority objective of the avoidance path is that it should be safe from any risk of collision. The path should also be the shortest by having the smallest turning radius or largest curvature, as known from Property 1-i. When a vehicle travels along a straight road and a static obstacle is initially located far from the vehicle by avoidance distance (cf. Fig. 3(a)), then, Problem A can be defined as follows.

Problem A: h-CCP for Avoidance Mode: When the initial pose is $\boldsymbol{p}_{i}\left(x_{i}, y_{i}, \theta_{i}^{\star}, \kappa_{i}^{\star}\right)$ and the meeting pose is $\boldsymbol{p}_{m}\left(x_{m}, y_{m}, \theta_{m}, \kappa_{m}^{\star}\right)$, find a continuous curvature path with minimum lateral acceleration (or maximum comfort), where $\theta_{i}^{\star}=\frac{\pi}{2}$ and $\kappa_{i}^{\star}=\kappa_{m}^{\star}=0$ in the local coordinate, cf. Fig. 1(a) (the superscript $\star$ refers to known information from geometric constraints).

Since the boundary condition for $x_{i}, y_{i}, x_{m}, y_{m}$ and $\theta_{m}$ is not fully given and thus it causes the algorithmic procedure to have too many variables to iterate, additional constraints are required to obtain an unique solution.

Here, two kinds of constraints are implemented, with one being the geometric safety constraint and the other being the solution constraint. As the geometric safety constraint, the boundary condition at the meeting pose $\boldsymbol{p}_{\boldsymbol{m}}$ should be specified. Since the obstacle boundary is modeled by a circle, the $\boldsymbol{p}_{\boldsymbol{m}}$ is located at the point around the circle so as to assign the path to make the closest turning, which results in the shortest travel length while also assuring safety i.e., Property 1-i. At this point, the orientation $\theta_{m}$ at $\boldsymbol{p}_{\boldsymbol{m}}$ becomes a design parameter such that the point around the obstacle circle (i.e., meeting position $x_{m}, y_{m}$ ) can be determined from the tangential line $\ell_{m}$ on the circular boundary as its slope $\tan \left(\theta_{m}\right)$ 
(cf. Fig. 9(a) presents the case that avoids and passes the obstacle on its right side).

Under the geometric safety constraint, this problem can be solved by a composition of two clothoids and its parametric iterations [32], [34]. The two clothoids are composed as follows. The first clothoid, $C_{1}$ is generated from its origin to end with the deflection $\delta_{1}$ (>0 from Eq.(5)), $\kappa_{1}$ and $\theta_{1}$ $\left(=\frac{\pi}{2}-\delta_{1}\right)$ by a constant $\alpha_{1}$. The second clothoid, $C_{2}$ is generated in the same way as $C_{1}$, but it is rotated and translated for its end to meet the end of $C_{1}$ while satisfying $G^{1}$ and $G^{2}$ continuity, i.e., by making the ending pose of $C_{1}$ as well as of $C_{2}$. A pair of clothoids which satisfy both of the boundary conditions is a solution.

The other constraint is to minimize the lateral acceleration. The lateral acceleration affects the passenger ride comfort with the proportionality of the curvature, thus it needs to minimize the maximal curvature as much as possible. Here, it is evident that the maximal curvature is correspondent to the maximal sharpness due to linear scalability as Eq.(1) and we focus on the sharpness because it is intended to formulate the iterative procedure by varying the sharpness under geometric constraints. The following optimization rule is imposed in order to formulate the minimization of the maximal curvature.

$$
\begin{aligned}
& \underset{\hat{\alpha}}{\operatorname{minimize}} \max _{\alpha_{i} \in C_{i}}\left[\alpha_{1}, \alpha_{2}\right]: \wp_{\mathbf{s}} \\
& \text { subject to } \kappa \leq \bar{\kappa}_{\text {limit }}, \alpha \leq \bar{\alpha}_{\text {limit }}, \quad\left(s \in\left[s_{i}, s_{m}\right]\right),
\end{aligned}
$$

where $\hat{\alpha}$ is the maximum sharpness among $\alpha_{1}, \alpha_{2}$.

This optimized solution corresponds to the performance measure, where $\wp_{s}$ corresponds to the speed for the human arms, thus the solution minimizes the maximum speed of the human arms during the avoidance. The rate of steering angle rotation is limited by mechanical or actuator limits as $\bar{\kappa}_{\text {limit }}$ and $\bar{\alpha}_{\text {limit }}$.

In Fig.9(a), it is shown how two clothoids are composed with parametric variation under geometric constraints. In the figure, $\theta_{m}$ is initially assumed, then $\boldsymbol{p}_{\boldsymbol{m}}$ is positioned on $\ell_{m}$ by geometric safety constraint. Two clothoids $C_{1}$ and $C_{2}$ are composed to have both end points as $\boldsymbol{p}_{\boldsymbol{o}}$ and $\boldsymbol{p}_{\boldsymbol{o}}^{\prime}$, and the resultant segment is shifted for $\boldsymbol{p}_{\boldsymbol{o}}^{\prime}$ to be equal to $\boldsymbol{p}_{\boldsymbol{m}}$ with the same orientation of the line $\ell_{m}$.

By the way, there are numerous feasible solutions which satisfy given boundary condition in Problem A, thus following solution constraint is imposed; MSC: Minimax Sharpness Constraint. It finds a pair of clothoids having the minimum of maximal sharpness value for each pair of clothoids. The MSC constrains two sharpness values to be same. Both clothoids have the same sharpness value of $\alpha_{1}=\alpha_{2}{ }^{7}$

Thus, as $\alpha_{1}$ (or $\alpha_{2}$ ) increases, the end point $\boldsymbol{p}_{\boldsymbol{o}}$ varies in the direction of the dotted arrow $(\nearrow)$ in Fig.9(a). A convergence criteria for this variation is formulated by $D_{\alpha}$, the horizontal shortest distance between $\boldsymbol{p}_{\boldsymbol{o}}$ and $\ell_{i}$. For $\boldsymbol{p}_{\boldsymbol{o}}$ to be sufficiently closer to $\boldsymbol{p}_{\boldsymbol{i}}$ within a given threshold, $\theta_{m}$ is also varied as well where $D_{\alpha}$ (the vertical distance between $\boldsymbol{p}_{\boldsymbol{o}}$ and $\boldsymbol{p}_{\boldsymbol{i}}$ ).

Eq.(9) presents the rules for parameter variation and its convergence (Iteration \#1), where the parameter $\alpha$ is iteratively varied until the corresponding determinant function $D_{\alpha}$

\footnotetext{
${ }^{7}$ For more details about MSC, please refer to [33].
}

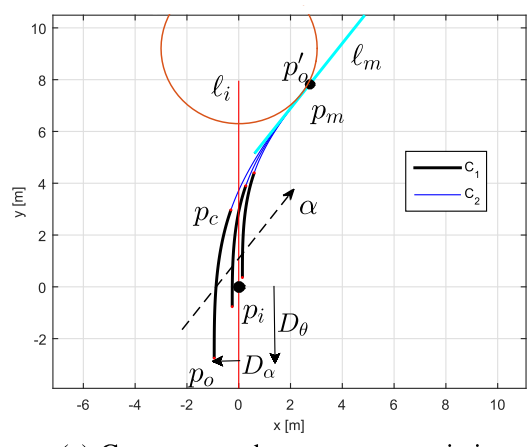

(a) Convergence by parameter variation

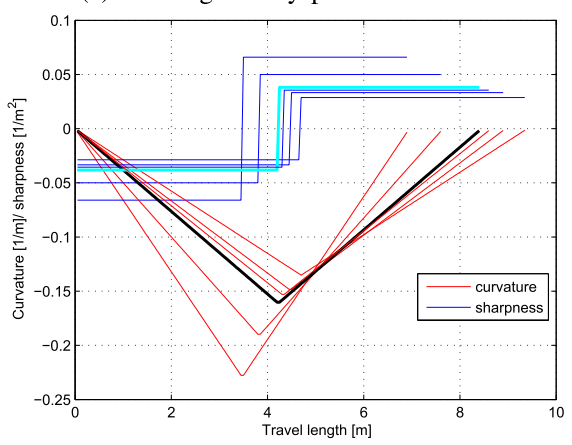

(b) C-S diagram

Fig. 9. Problem A solution result.

becomes smaller than some threshold. The parameter $\alpha$ is halved when the sign of $D_{\alpha}$ changes, i.e., the iteration for the convergence rule is the same as the bisection method [38].

$$
\begin{aligned}
\alpha & =\alpha+|\partial \alpha| \cdot \operatorname{sgn}\left(D_{\alpha}\right), \\
D_{\alpha} & =x_{o}-x_{i}, \\
\partial \alpha & = \begin{cases}\frac{\partial \alpha}{2}, & \text { if } D_{\alpha} \cdot D_{\alpha}^{\prime}<0, \\
\partial \alpha, & \text { otherwise, }\end{cases}
\end{aligned}
$$

where, $x_{o}$ is the $x$-coordinate value of $\boldsymbol{p}_{\boldsymbol{o}}$ and $\operatorname{sgn}(\cdot)$ is the sign function, which could be defined by a positive or negative value, and $D_{\alpha}^{\prime}$ is $D_{\alpha}$ at the previous iteration. In addition, $\partial$ imposes a differential change of the following variable, where its initial value is assumed in the algorithm.

$$
\begin{aligned}
& \theta_{m}=\theta_{m}+|\partial \theta| \cdot \operatorname{sgn}\left(D_{\theta}\right), \\
& D_{\theta}=y_{o}-y_{i}, \\
& \partial \theta= \begin{cases}\frac{\partial \theta}{2}, & \text { if } D_{\theta} \cdot D_{\theta}^{\prime}<0, \\
\partial \theta, & \text { otherwise }\end{cases}
\end{aligned}
$$

where $y_{o}$ is the $y$-coordinate value of $\boldsymbol{p}_{\boldsymbol{o}}$.

Eq.(10) presents Iteration $\mathbf{\# 2}$ for $\theta_{m}$ which is similar to Eq.(9). The convergence criteria for both iteration procedures are defined as follows and as shown in Fig.9(a).

$$
\begin{aligned}
D_{\alpha} & <\varepsilon_{\alpha}, \\
D_{\theta} & <\varepsilon_{\theta},
\end{aligned}
$$

where $10^{-3} \mathrm{~m}$ is applied to make fast convergence with accuracy, $\varepsilon_{\alpha}$ and $\varepsilon_{\theta}$ are threshold distance in [m] for $\alpha$ and 


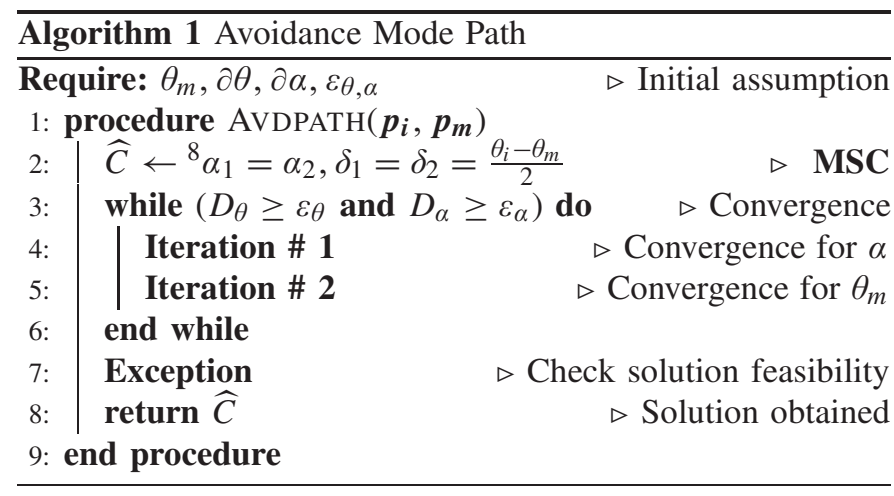

$\theta$ variation respectively. In addition, the determinant function $D_{\alpha}$ is defined as the difference between $\boldsymbol{p}_{\boldsymbol{o}}$ and $\boldsymbol{p}_{\boldsymbol{i}}$ (or $\ell_{i}$ ) along $x$-axis, and $D_{\theta}$ is the difference between $\boldsymbol{p}_{\boldsymbol{o}}$ and $\boldsymbol{p}_{\boldsymbol{i}}$ along $y$-axis.

The algorithmic procedure for the avoidance mode (Algorithm 1) is described as follows. First, the parameter $\theta_{m}$ is assumed (initially $\frac{\pi}{4}$ ), then $\boldsymbol{p}_{\boldsymbol{m}}$ on the obstacle boundary is determined by the tangential line $\ell_{m}$. Next, the composed clothoid $\widehat{C}$ (cf. Fig. 9(a)) is translated for its end to $\boldsymbol{p}_{\boldsymbol{m}}$, then a solution using MSC is obtained with $\boldsymbol{d}_{\boldsymbol{a v d}}$ using Iteration \#1. If the current position of the vehicle differs from $\boldsymbol{d}_{\boldsymbol{a v d}}$, the parameter $\theta_{m}$ is iteratively varied until the distance between the obstacle and the vehicle is close enough to $\boldsymbol{d}_{\boldsymbol{a v d}}$ within a threshold $\varepsilon=10^{-3}[\mathrm{~m}]$ by Iteration \#2.

If the solution has its maximum (or minimum) curvature or sharpness over $\bar{\kappa}_{\text {limit }}$ or $\bar{\alpha}_{\text {limit }}$, and if the condition of too close obstacle position as $\theta_{m}<0$ is reached, then the obtained path is not feasible. In that case, it remains that the only way to reduce the vehicle speed is to stop (cf. Exception in line 7 of Algorithm 1). Algorithm 1 describes the procedure used to obtain the proposed solution.

From the pattern of avoidance distance acquired from Exp.2, i.e., Property 1-ii and Property 1-iii, the desirable vehicle speed is taken from the relation as Eq.(6), such that the obtained $\boldsymbol{d}_{\boldsymbol{a v d}}$ could give a desirable vehicle speed to enter into the avoidance mode. Thus, if the current vehicle speed is over the calculated desirable vehicle speed, the vehicle should reduce its speed to the given value until the avoidance begins.

A schematic example for Problem A is shown in Fig. 9(a), the curvature/sharpness diagram $(C-S$ diagram $)$ for the obtained solution is depicted in Fig. 9(b).

As shown in Fig. 9(a), two clothoids $C_{1}$ and $C_{2}$, are generated with given parameters $\alpha_{1,2}, \kappa_{1,2}$ and $\delta_{1,2}$ where the subscripts $(1,2)$ present the parameters of both clothoids as the same (cf. Algorithm 1: line 2), from $\boldsymbol{p}_{\boldsymbol{o}}, \boldsymbol{p}_{\boldsymbol{o}}^{\prime}$ to $\boldsymbol{p}_{\boldsymbol{c}}$ respectively, to be composed as $\widehat{C}$. By independently varying $\theta_{m}$ and $\alpha_{1,2}$, the algorithm finds the feasible clothoids pair of $C_{1}, C_{2}$ that satisfies both boundary conditions of $\boldsymbol{p}_{\boldsymbol{i}}$ and $\boldsymbol{p}_{\boldsymbol{m}}$, while being tangential to $\ell_{i}$ and $\ell_{m}$, respectively (cf. Fig. 9(a)). The solution is obtained after convergence passes both of the conditions of $D_{\alpha}$ and then, $D_{\theta}$. It is also clear that the solution path is generated under $\ell_{m}$ line and the obstacle boundary is

\footnotetext{
$8 \leftarrow$ indicates that two clothoids are composed by given parameters.
}

located in the upper side of $\ell_{m}$, so the vehicle motion along the path is considered to be safe from the risk of collision.

The corresponding C-S diagrams are shown in Fig. 9(b). Among the feasible solutions that have passed the $D_{\alpha}$ and the $D_{\theta}$ condition, a solution satisfying MSC is chosen as being marked by a black-bold line in curvature and a cyan-bold line in sharpness (cf. Fig. 9(b)).

\section{B. Problem B}

This subsection solves Problem B for the recovery mode. After the obstacle is avoided, for instance, once it is behind the vehicle, the driver steers the vehicle to make the transition to the other free lane (lane change) while recovering its initial orientation with zero curvature or zero steering angle. The initial pose $\boldsymbol{p}_{\boldsymbol{m}}$ (the final pose of Problem A) is given but, the final pose is not fully known where only the lateral distance to be recovered is given by the lane width constraints. Thus, Problem $\mathbf{B}$ is defined as follows.

Problem B: h-CCP for Recover Mode: When the initial pose is $\boldsymbol{p}_{m}\left(x_{m}^{\star}, y_{m}^{\star}, \theta_{m}^{\star}, \kappa_{m}^{\star}\right)$ and the final pose is $\boldsymbol{p}_{f}\left(x_{f}, y_{f}, \theta_{f}^{\star}, \kappa_{f}^{\star}\right)$, find a $\mathbf{C C P}$ solution which minimizes steering work under given maximal curvature and sharpness, where $\kappa_{m}^{*}=\kappa_{f}^{*}=0, \theta_{f}^{*}=\frac{\pi}{2}$ in the local coordinate, cf. Fig. 1(a) (the superscript $\star$ refers to known information from the solution of problem $A$ and boundary constraint).

As described in Section II, the steering pattern for the recovery mode displays a less sharp, looser, and flatter shape than the avoidance mode. This steering pattern implies that the human driver operates to minimize his/her steering work under the allowable lateral acceleration during the recovery mode, thus it could be emulated by a similar shaped curvature diagram that has a lower peak and longer travel length than that of avoidance mode. For the curvature diagram design, the solution constraint for Problem B could be formulated with two performance measures to be optimized under constraints as follows; steering rotation $\left(\wp_{\mathbf{r}}\right)$ and steering speed $\left(\wp_{\mathbf{s}}\right)$.

$$
\begin{array}{ll}
\underset{\kappa(s)}{\operatorname{minimize}} & \int_{s_{m}}^{s_{f}}|\alpha(s)| d s: \wp_{\mathbf{r}} \\
\underset{\hat{\alpha}}{\operatorname{minimize}} & \max _{\alpha_{i} \in C_{i}} \widehat{C}\left(\alpha_{3}, \alpha_{4}\right): \wp_{\mathbf{s}} \\
\text { subject to } \kappa \leq \bar{\kappa}_{\text {max }}, \alpha \leq \bar{\alpha}_{\text {max }}, \quad\left(s \in\left[s_{m}, s_{f}\right]\right)
\end{array}
$$

where, $\kappa_{\max }$ is given by the allowable lateral acceleration and vehicle speed as $a_{\max }=\kappa_{\max } v^{2}(v$ : vehicle speed $[\mathrm{m} / \mathrm{s}])$ and ISO 2631-1 comfort measure, e.g., for Not uncomfortable $a_{\max } \leq 0.315 \mathrm{~m} / \mathrm{s}^{2}$.

Eq.(13) describes the solution constraint of minimizing the amount of steering rotation and the maximum steering speed matched to the amount of curvature variation and maximum sharpness, respectively. The solution could be obtained by simultaneously minimizing $\wp_{\mathbf{r}}$ and $\wp_{\mathbf{s}}$ by adjusting the clothoids parameters. By the way, since the vehicle speed is constant through the overall lane change maneuvering, the constraints $\kappa_{\max }$ and $\alpha_{\max }$ of Problem $\mathbf{B}$ are always below the values of the obtained solution in Problem A. Thus, it is derived that the minimax sharpness of this problem is 


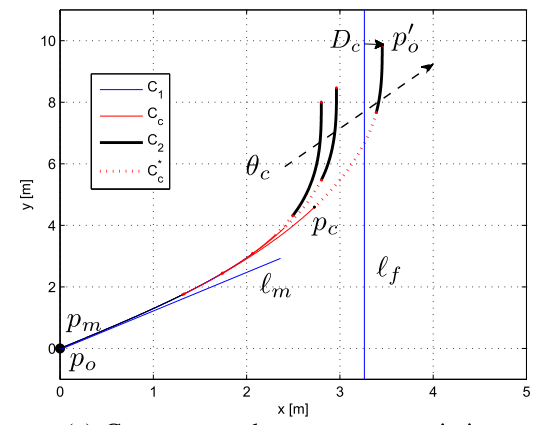

(a) Convergence by parameter variation

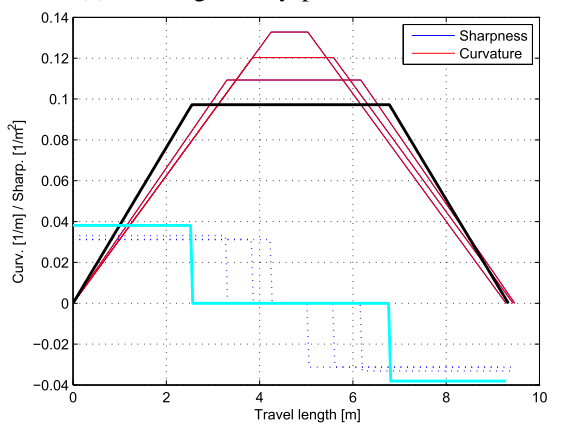

(b) C-S diagram

Fig. 10. Problem B solution results.

constrained to the sharpness of the solution of Problem A, i.e., $\alpha_{3,4} \leq \alpha_{1,2}$, the clothoids composition is similar to Problem A, except the condition that the lateral distance to be arrived is longer than the lateral distance avoided as $\left(x_{m}-x_{i}\right) \leq\left(x_{f}-x_{m}\right)$, thus the composed path requires an additional arc segment. In order to explain this geometric compromise, the following example is described with Fig. 10.

With the given boundary condition $\boldsymbol{p}_{\boldsymbol{m}}$, the end position of the composed segment $\boldsymbol{p}_{\boldsymbol{o}}^{\prime}$ should be sufficiently close to the line $\ell_{f}$ within a threshold. The symmetric clothoids $C_{1}, C_{2}$ and the symmetric $\operatorname{arcs} C_{c} C_{c}^{*}$ are composed with each other under the orientation continuity constraint. The parameter $\theta_{c}$ is the angle of the corresponding circumference for $C_{c}$ or $C_{c}^{*}$ (cf. Appendix) and, as $\theta_{c}$ increases, the end point $\boldsymbol{p}_{\boldsymbol{o}}^{\prime}$ expands outward in the direction of the dotted arrow $(\nearrow)$ as shown in Fig. 10(a).

In Fig. 10, the solution path and its C-S diagram for the problem $\mathbf{B}$ example are shown where $\boldsymbol{p}_{\boldsymbol{m}}\left(0,0, \frac{\pi}{6}, 0\right)$, $\boldsymbol{p}_{f}\left(3, y_{f}, \frac{\pi}{2}, 0\right)$, and the solution includes two symmetric clothoids with additional arc segments. Each solution path in Fig. 10(b) satisfies the boundary conditions at both ends, but the sharpness and maximum curvature value differs from those of the other solutions. Among the solution candidates obtained, first, the optimized solution is to select the path with the smallest maximum curvature value $\kappa_{\max }$. One can observe in Fig. 10(b) that the steering rotation $\left(\wp_{\mathbf{r}}\right)$ decreases as the length of the arc segment becomes longer, since $\int_{s_{a}}^{s_{b}}|\alpha(s)| d s=0$ for the arc segment $s \in\left[s_{a}, s_{b}\right]$. Simultaneously, it can be noticed that the maximum sharpness $\left(\wp_{\mathbf{s}}\right)$ of the composed path increases as the arc segment expands. Thus, both performance measures are minimized until the sharpness of Problem B increases to the sharpness of Problem A, which is denoted in Fig. 10(b) by the black-bold line (curvature) and the cyan-bold line (sharpness).

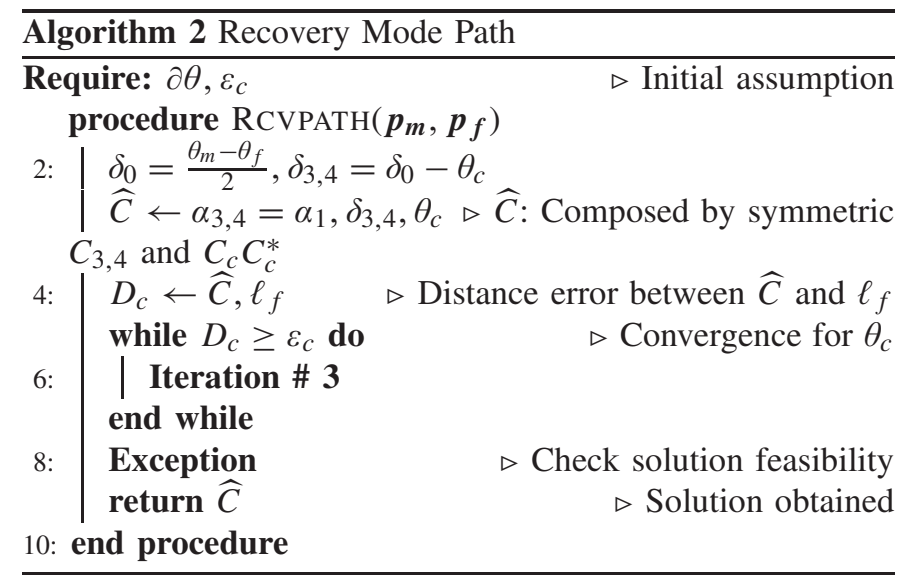

As shown in Algorithm 2, the sharpness $\alpha_{3,4}$ (for the two clothoids $C_{3}$ and $C_{4}$ in the recovery mode path) are all the same as the sharpness $\alpha_{1,2}$ obtained in Problem $\mathbf{A}$ and the deflections $\delta_{3,4}$ are initially set to be $\delta_{0}$ and varied by $\delta_{c}$ subtraction (line 2). $\widehat{C}$ is composed of two clothoids, $C_{3}$ and $C_{4}$ and symmetric two circular arcs $C_{c} C_{c}^{*}$. The only variable for iterative convergence is the internal angle $\theta_{c}$ for the circular arc, which determine the deflections of $\delta_{3,4}$ (line 3 ).

$$
\begin{aligned}
\theta_{c} & =\theta_{c}+|\partial \theta| \cdot \operatorname{sgn}\left(D_{c}\right), \\
D_{c} & =x_{t g}-x_{o}^{\prime}, \quad\left(x_{t g}=x_{f}-x_{m}\right) \\
\partial \theta & = \begin{cases}\frac{\partial \theta}{2}, & \text { if } D_{c} \cdot D_{c}^{\prime}<0, \\
\partial \theta, & \text { otherwise }\end{cases}
\end{aligned}
$$

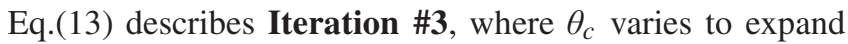
the arc portion in $C_{3}$ and $C_{4}$. By adjusting the arc portion, the end point $\left(p_{o}^{\prime}\right)$ of the composed clothoid $(\widehat{C})$ could reach the target line $\ell_{f}$ within a threshold (cf. Fig. 10(a)). The convergence criteria is similar to Iteration \#1, \#2 as follows.

$$
D_{c}<\varepsilon_{c},
$$

where $\varepsilon_{c}$ could be $10^{-3} \mathrm{~m}$. Note that for the path resulting in $\theta_{c} \leq 0$, Iteration $\mathbf{\# 3}$ in this algorithm is not effective for the given boundary condition, and it needs to be solved by Iteration \#1 so as to find a new $\alpha_{3,4}$. The feasibility checking for the obtained solution is also performed in the Exception stage (line 8) whether the obtained solution exceeds given limits such as $\bar{\kappa}_{\max }, \bar{\alpha}_{\max }$ as given in Eq.(8).

\section{Examples of Complete Lane Change Maneuvering Based on $h-C C P$}

From the two defined sub-problems and the obtained solutions, the h-CCP is generated with the corresponding C-S diagram. The h-CCP has a total of four clothoids and its curvature diagram has two different convex ( $C$-shaped) curves of the avoidance and recovery modes. The proposed solution is applied to two lane change maneuvering examples, where the first example is for a large obstacle (passenger vehicle) of radius $r_{d}=4 \mathrm{~m}$ with a lateral distance of $6 \mathrm{~m}$, and the second example is the strict lane change for a small obstacle (bicycle or people) of $r_{d}=1.6 \mathrm{~m}$ with a lateral distance of $3.5 \mathrm{~m}$. 


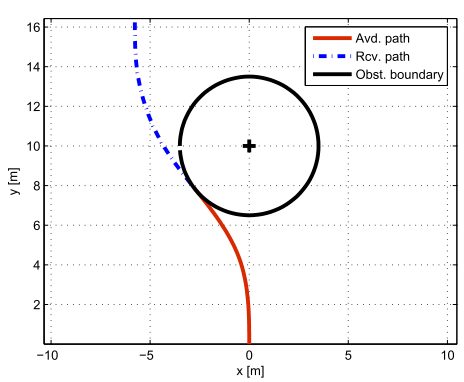

(a) Path for large obstacle (Ex. I)

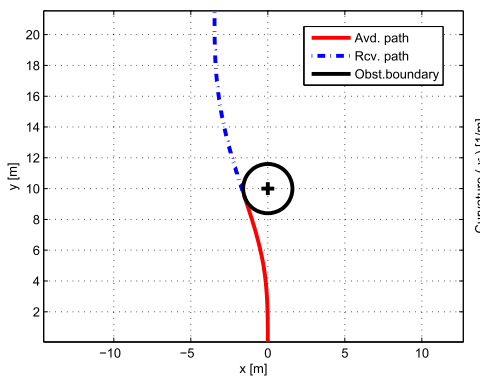

(c) Path for small obstacle (Ex. II)

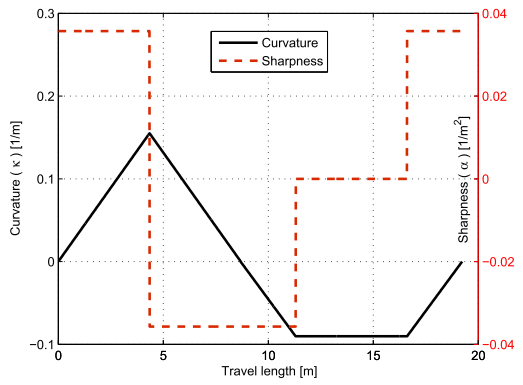

(b) C-S diagram (Ex. I)

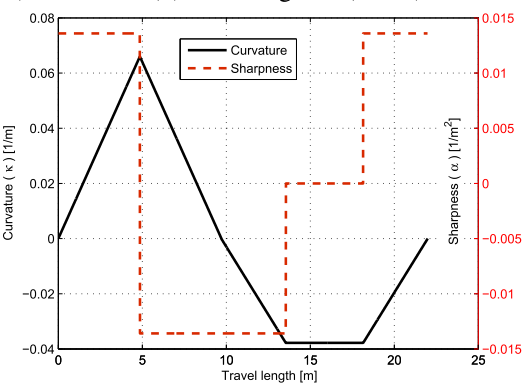

(d) C-S diagram (Ex. II)

Fig. 11. h-CCP of obstacle avoidance using lane change maneuver.

In the results shown in Fig. 11, two examples, Ex. I and II are resolved by tackling two different types of paths (avoidance/recovery modes), and both obtained paths successfully avoid the obstacle while taking a continuous curvature along the travel length as shown in Fig. 11(b) and Fig. 11(d). The proposed algorithms implement the human driving patterns and benefit from the reduced number of iterations by restricting the variable parameters while simultaneously assuring the convergence. Furthermore, the algorithms cope with the different obstacle sizes/positions and lane width in spite of the incomplete boundary conditions. The algorithmic efficiency is analyzed from a representative graph shown in Fig. 12.

Fig. 12(a) shows the results of the variation of travel length $s$ (scaled by $\frac{1}{50}$ ), the maximum curvature $\left(\kappa_{\max }\right)$, and the maximum sharpness $\left(\alpha_{\max }\right)$ according to the $\theta_{m}$ increase (cf. Problem A and Algorithm 1), where the solution converges to $\alpha_{\max }$ and $\kappa_{\max }$ by minimizing $\wp_{s}$ in Eq.(8) and satisfies the $\boldsymbol{d}_{\text {avd }}$ constraint by increasing $s$ (the converged $\theta_{m}$ is $\left.51.45[\mathrm{deg}]\right)$. Fig. 12(b) shows an example of the algorithmic convergence $D_{\theta}$ to the given error bound of $0.01 \mathrm{~m}$ within 10 iterations, which estimates about $150 \mathrm{~ms}$ (with MATLAB and Pentium Dual Core $1.90 \mathrm{GHz}$ ). We omit the result for recovery mode path since it exhibits very similar performances to that of the avoidance mode path.

\section{Evaluation of THE h-CCP}

In this section, the obtained path is compared with other lane change methods. In order to evaluate the performance of the proposed solution, five representative path generation methods, such as the quintic polynomials [5], Bézier spline [8], [9], [39], Dubins path, FS path and Wilde path are obtained and compared. All of the paths should keep the lateral width for lane change; as well as avoid the given obstacle within an error bound (e.g., $10^{-3} \mathrm{~m}$ ). Thus, each path is

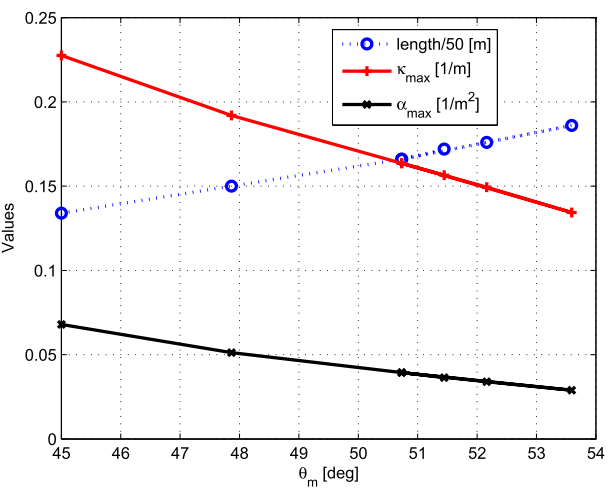

(a) Path variation by $\theta_{m}$

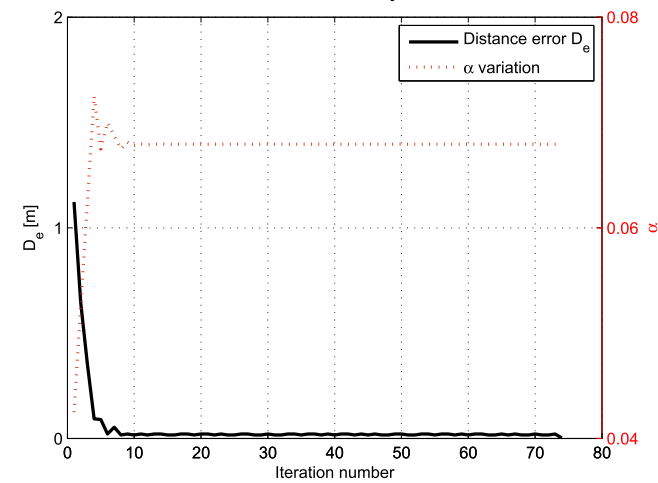

(b) Iterative convergence

Fig. 12. Algorithmic convergence for avoidance mode path.

iteratively obtained under those constraints. Fig. 13 depicts for the comparison of the obtained path with other methods for example I.

As shown in Fig. 13(a), all of the obtained paths avoid the given obstacle (denoted by Obst. Bnd., i.e., obstacle boundary) and arrive at the other lane, and even if all of the 


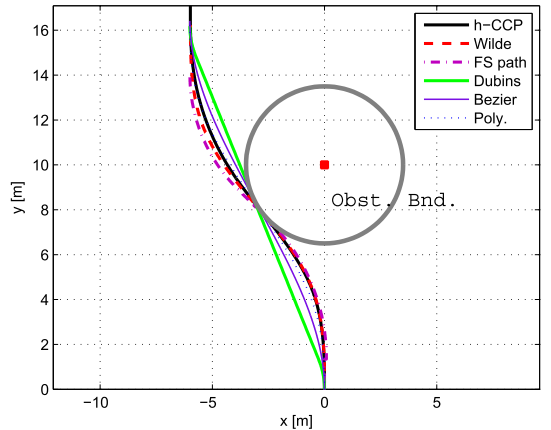

(a) Paths

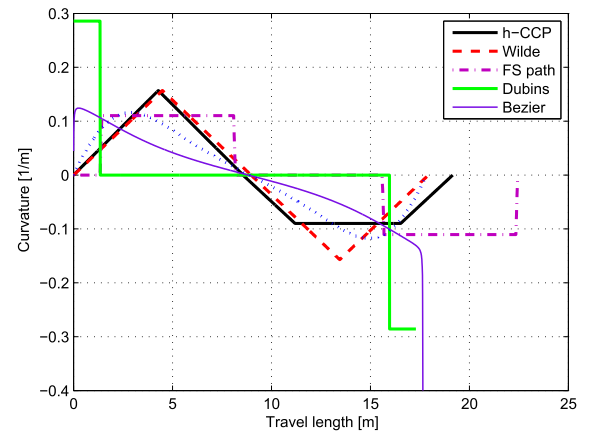

(b) Curvatures

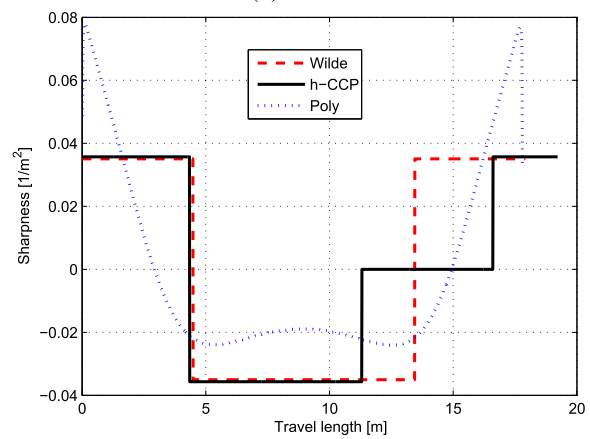

(c) Sharpnesses

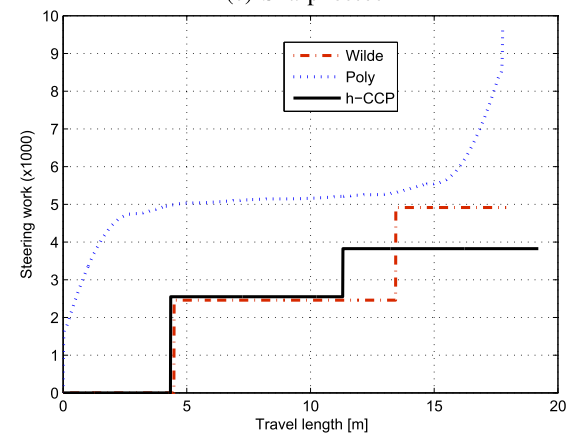

(d) Steering works

Fig. 13. Comparison of the proposed h-CCP with other paths.

paths are geometrically smooth in the position and orientation domain $\left(G^{0}\right.$ and $\left.G^{1}\right)$, they are largely different in the curvature domain $\left(G^{2}\right)$ as shown in Fig. 13(b). In particular, the curvature of FS path ${ }^{9}$; and the Dubins and Bézier paths have much higher peaks or drastic changes in the curvatures, i.e. discontinuities; thus, these three paths are not regarded in further diagrams.

\footnotetext{
${ }^{9}$ The mechanical limit $\bar{\kappa}_{\text {limit }}=0.489$ and $\bar{\alpha}_{\text {limit }}=1.227$ are used. (cf. Eq.(8)) since FS path uses the curvature limit for the clothoid generation [17].
}

TABLE III

Performance Evaluation of the Proposed Path ([m], $[\mathrm{rad}])$

\begin{tabular}{|c|c|c|c|c|c|c|c|}
\hline path & length & $\mathcal{W}_{s t}$ & $\kappa_{\max }$ & $\kappa_{\min }$ & $\alpha_{\max } \alpha_{\min }$ & $\tau_{\max }$ & $\wp_{r}$ \\
\hline Poly & 17.79 & 0.0097 & 0.1175 & -0.1163 & 0.0241 -0.078 & 2.6871 & 0.4607 \\
\hline Bézier & 17.65 & 12.6924 & 0.1243 & -0.3999 & $3.5488-17.8001$ & 1422.7 & 0.6128 \\
\hline Dubins & 17.30 & 1632.7 & 0.2857 & -0.2857 & $-\infty$ & 2857.14 & 0.5753 \\
\hline$\overline{\text { FS }}$ & 22.43 & 5.6430 & 0.1104 & -0.1104 & $1.227-1.227$ & 122.67 & 0.4893 \\
\hline Wilde & 17.92 & 0.0049 & 0.1570 & -0.1570 & $0.0351-\mathbf{0 . 0 3 5 1}$ & 7.0101 & 0.5753 \\
\hline h-CCP & 19.16 & 0.0039 & 0.1568 & -0.0898 & $0.0366-0.0366$ & 7.3266 & 0.4893 \\
\hline
\end{tabular}

Then, aside from those three paths, the sharpness diagrams are shown in Fig. 13(c). All of the paths can not be shown in the same scale due to much high peaks in those three paths.

In the sharpness diagrams, the results of the obtained h-CCP, Wilde and Poly paths are compared, where the segment based methods (h-CCP, Wilde path) have simple and steady curvature, but the Poly path has greater fluctuations at boundary conditions. As mentioned in [30], the Wilde path has minimum sharpness and curvature in an effort to be human natural and easily drivable.

The h-CCP also shows almost the same $\kappa_{\max }$ but a smaller $\kappa_{\min }, \mathcal{W}_{s t}$ (cf. Eq.(7)) than the Wilde path. Fig. 13(d) highlights the steering work comparison for the three paths. As shown in the figure, Poly path increases the steering work more than other methods up to the double at the end. For the hCCP and the Wilde path, the proposed h-CCP shows smaller $\mathcal{W}_{s t}$ than the Wilde path by $-20 \%$. Qualitative measures for the above results are presented in Table III.

As shown in Table III, the items of length and $\tau_{\max }$ signify the travel length, and the maximum difference for $\alpha$ change respectively. The superior performance for each item is marked in bold. The Poly path is superior to other methods by the smallest $\tau_{\max }$ and $\wp_{\mathbf{r}}$; however, it has larger $\alpha_{\min }$ and $\kappa_{\min }$ and $\mathcal{W}_{s t}$ than the proposed path. Although the Dubins, FS, or Bézier paths obtain higher performance in specific items, the curvature or sharpness diagrams indicate that they are not appropriate for steering control due to the drastic change of the sharpness $\left(\alpha_{\min }\right)$ or to the required big steering torque $\left(\tau_{\max }\right)$.

The proposed path has superior performance in the sharpness and curvature as Wilde path and spends small $\wp_{s}$ close to the Poly path. Furthermore, the proposed path records the smallest value of $\left|\kappa_{\min }\right|$ which is proportional to the lateral acceleration or deterioration of ride comfort. Consequently, it is obvious that the proposed path is smooth and simple in terms of curvature and sharpness such that the steering control follows the path without complexity, and it also ensures the safety for obstacle avoidance with minimal magnitude of steering rate (or sharpness) as well as minimal steering work for recovery mode; thus, it provides natural and human favorable maneuvering. Note that if the vehicle speed is constant through the lane change maneuver, the comfort measure depends on $\kappa_{\max }$ and $\boldsymbol{v}_{\text {avd }}$ (cf. Eq.(6)).

\section{CONClusion}

This paper presents a continuous curvature path generation method inspired by human driving patterns (h-CCP) performing lane change maneuvers for obstacle avoidance. Based on the analysis of human driving data, two different driving 


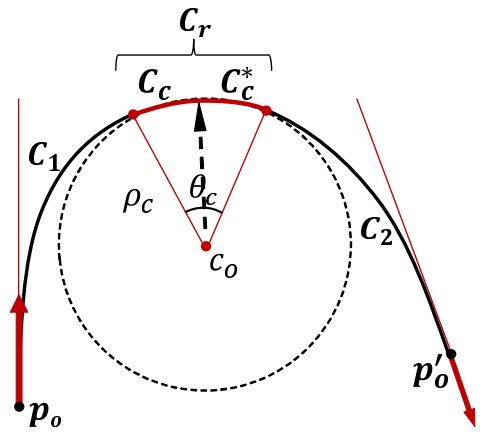

Fig. 14. Clothoids composition with symmetric arcs.

modes for static obstacle avoidance are found, and those human patterns are implemented in order to formulate the path generation problem of lane change maneuvering. The problem is then defined specifically with additional constraints so as to be solved using efficient iterative algorithms. As compared to the other smooth paths defined in the literature, the proposed h-CCP shows noticeably enhanced ride comfort and minimized steering work, similar to driving as it would be performed by experienced human drivers. As found by the planned experiments, the steering rate or sharpness could be an important parameter that reflects the environmental status for the vehicle and it also represents human feeling as well as ride comfort while following the given path. In this respect, the path generation by separating two modes is efficient to consider both human aspect and algorithmic efficiency. Furthermore, the proposed methodology could be expanded by representing environmental status with risk measures in order to provide the passengers in the vehicle with human natural and favorable maneuvering.

The proposed path is not the best solution in mathematical or performance point of view, but a better computational solution which is closer to human driver's pattern and considers comfort. In future work, huge amounts of human driving data mining by big data treatment and solution updates with machine learning could enrich its practical applicability and generality to various kinds of vehicles and passengers.

\section{APPENDIX}

\section{Clothoids Composition in Problem B}

In Fig.14, two clothois $C_{1}$ and $C_{2}$ are connected with arc segments $C_{c}$ and $C_{c}^{\prime}$, which has same curvature (or same radius of curvature). A composed pair of symmetric $C_{c}$ and $C_{c}^{\prime}$ is named as $C_{r}$ which has circumferential angle $\theta_{c}$ where the circle has its center $C_{o}$ with the radius $\rho_{c}$.

\section{REFERENCES}

[1] L. Adouane, Autonomous Vehicle Navigation: From Behavioral to Hybrid Multi-Controller Architectures. Boca Raton, FL, USA: CRC Press, 2016

[2] X.-C. Lai, S. S. Ge, and A. Al Mamun, "Hierarchical incremental path planning and situation-dependent optimized dynamic motion planning considering accelerations," IEEE Trans. Syst., Man, Cybern. B, Cybern., vol. 37, no. 6, pp. 1541-1554, Dec. 2007.

[3] J.-B. Tomas-Gabarron, E. Egea-Lopez, and J. Garcia-Haro, "Vehicular trajectory optimization for cooperative collision avoidance at high speeds," IEEE Trans. Intell. Transport. Syst., vol. 14, no. 4, pp. 1930-1941, Dec. 2013.
[4] L. Adouane, "Reactive versus cognitive vehicle navigation based on optimal local and global PELC," Robot. Auto. Syst., vol. 88, pp. 51-70, Feb. 2017.

[5] W. Nelson, "Continuous-curvature paths for autonomous vehicles," in Proc. IEEE Int. Conf. Robot. Automat., vol. 3, May 1989, pp. 1260-1264.

[6] I. Paromtchik, P. Garnier, and C. Laugier, "Autonomous maneuvers of a nonholonomic vehicle," in Proc. 5th Int. Symp. Experim. Robotics, Experim. Robot., Barcelona, Spain, 1998, pp. 276-288.

[7] N. Montes, M. C. Mora, and J. Tornero, "Trajectory generation based on rational Bezier curves as clothoids," in Proc. IEEE Intel. Vehicles Symposium, Jun. 2007, pp. 505-510.

[8] J. Chen, P. Zhao, T. Mei, and H. Liang, "Lane change path planning based on piecewise Bezier curve for autonomous vehicle," in Proc. IEEE Int. Conf. Veh. Electron. Saf., Dongguan, China, Jul. 2013, pp. 17-22.

[9] D. Gonzalez, J. Perez, R. Lattarulo, V. Milanes, and F. Nashashibi, "Continuous curvature planning with obstacle avoidance capabilities in urban scenarios," in Proc. 17th Int. IEEE Conf. Intell. Transp. Syst. (ITSC), Qingdao, China, Oct. 2014, pp. 1430-1435

[10] L. Labakhua et al., "Smooth trajectory planning for fully automated passengers vehicles: Spline and Clothoid based methods and its simulation," in Informatics in Control Automation and Robotics (Lecture Notes Electrical Engineering), vol. 15, A. Cetto, Ed. 2008, pp. 159-182, doi: 10.1007/978-3-540-79142-3_14.

[11] J. E. Naranjo, C. Gonzalez, R. Garcia, and T. de Pedro, "Lanechange fuzzy control in autonomous vehicles for the overtaking maneuver," IEEE Trans. Intell. Transport. Syst., vol. 9, no. 3, pp. 438-450, Sep. 2008.

[12] J. Villagra, V. Milanés, J. Pérez, and J. Godoy, "Smooth path and speed planning for an automated public transport vehicle," Robot. Auto. Syst., vol. 60, no. 2, pp. 252-265, Feb. 2012, doi: 10.1016/j.robot.2011.11.001.

[13] M. Elbanhawi, M. Simic, and R. Jazar, "Randomized bidirectional BSpline parameterization motion planning," IEEE Trans. Intell. Transport. Syst., vol. 17, no. 2, pp. 406-419, Feb. 2016.

[14] K. Jo and M. Sunwoo, "Generation of a precise roadway map for autonomous cars," IEEE Trans. Intell. Transport. Syst., vol. 15, no. 3, pp. 925-937, Jun. 2014.

[15] L. E. Dubins, "On curves of minimal length with a constraint on average curvature, and with prescribed initial and terminal positions and tangents," Amer. J. Math., vol. 79, no. 3, pp. 497-516, 1957.

[16] J. Reeds and L. Shepp, "Optimal paths for a car that goes both forwards and backwards," Pacific J. Math., vol. 145, no. 2, pp. 367-393, Oct. 1990.

[17] T. Fraichard and A. Scheuer, "From reeds and Shepp's to continuouscurvature paths," IEEE Trans. Robot., vol. 20, no. 6, pp. 1025-1035, Dec. 2004.

[18] P. Symonds, R. S. Sharp, and D. Casanova, "A mathematical model for driver steering control, with design, tuning and performance results," Vehicle Syst. Dyn., vol. 33, no. 5, pp. 289-326, May 2000.

[19] C. C. Macadam, "Understanding and modeling the human driver," Vehicle Syst. Dyn., vol. 40, nos. 1-3, pp. 101-134, Jan. 2003.

[20] G. Markkula, O. Benderius, and M. Wahde, "Comparing and validating models of driver steering behaviour in collision avoidance and vehicle stabilisation," in Vehicle System Dynamics. Oxford, U.K.: Taylor and Francis, 2014, pp. 1-24.

[21] J. Edelmann, M. Plöchl, W. Reinalter, and W. Tieber, "A passenger car driver model for higher lateral accelerations," Vehicle Syst. Dyn., vol. 45, no. 12, pp. 1117-1129, Dec. 2007.

[22] P. Petrov and F. Nashashibi, "Adaptive steering control for autonomous lane change maneuver," in Proc. IEEE Intell. Vehicles Symp., Gold Coast, QLD, Australia, 2013, pp. 835-840.

[23] T. Tsuji, Y. Tanaka, P. G. Morasso, V. Sanguineti, and M. Kaneko, "Biomimetic trajectory generation of robots via artificial potential field with time base generator," IEEE Trans. Syst., Man Cybern., C, Appl. Rev., vol. 32, no. 4, pp. 426-439, Nov. 2002.

[24] N. Yuhara and J. Tajima, "Advanced steering system adaptable to lateral control task and Driver's intention," Vehicle Syst. Dyn., vol. 36, nos. 2-3, pp. 119-158, Sep. 2001.

[25] G. Bevan, H. Gollee, and J. O'reilly, "Automatic lateral emergency collision avoidance for a passenger car," Int. J. Control, vol. 80, no. 11, pp. 1751-1762, Nov. 2007.

[26] M. Yamakado and M. Abe, "An experimentally confirmed driver longitudinal acceleration control model combined with vehicle lateral motion," Vehicle Syst. Dyn., vol. 46, no. 1, pp. 129-149, Sep. 2008. 
[27] P. Narayan, P. Meyer, and D. Campbell, "Embedding human expert cognition into autonomous UAS trajectory planning," IEEE Trans. Cybern., vol. 43, no. 2, pp. 530-543, Apr. 2013.

[28] R. Hayashi, J. Isogai, P. Raksincharoensak, and M. Nagai, "Autonomous collision avoidance system by combined control of steering and braking using geometrically optimised vehicular trajectory," Vehicle Syst. Dyn., vol. 50, no. sup1, pp. 151-168, Jan. 2012.

[29] M. Rothhämel, J. I. Jkema, and L. Drugge, "Influencing driver chosen cornering speed by means of modified steering feel," Vehicle Syst. Dyn., vol. 52, no. 4, pp. 522-538, Apr. 2014.

[30] D. K. Wilde, "Computing clothoid segments for trajectory generation," in Proc. IEEE/RSJ Int. Conf. Intell. Robots Syst., Oct. 2009, pp. 2440-2445.

[31] R. Attia, R. Orjuela, and M. Basset, "Combined longitudinal and lateral control for automated vehicle guidance," Vehicle Syst. Dyn., vol. 52, no. 2, pp. 261-279, Feb. 2014.

[32] S. Gim, L. Adouane, S. Lee, and J.-P. Derutin, "Parametric continuous curvature trajectory for smooth steering of the car-like vehicle," in Proc. Int. Conf. Intell. Auton. Syst., Padova, Italy, 2014, pp. 1327-1342.

[33] S. Gim, L. Adouane, S. Lee, and J.-P. Dérutin, "Clothoids composition method for smooth path generation of car-like vehicle navigation," J. Intell. Robotic Syst., vol. 88, no. 1, pp. 129-146, Oct. 2017.

[34] S. Gim, L. Adouane, S. Lee, and J.-P. Derutin, "Smooth trajectory generation with 4D space analysis for dynamic obstacle avoidance," in Proc. 11th Int. Conf. Informat. Control, Autom. Robot., Vienna, Austria, 2014, pp. 802-809.

[35] H. Yoshida, S. Shinohara, and M. Nagai, "Lane change steering manoeuvre using model predictive control theory," Vehicle Syst. Dyn., vol. 46, no. 1 , pp. 669-681, Sep. 2008.

[36] A. Chakravarthy and D. Ghose, "Obstacle avoidance in a dynamic environment: A collision cone approach," IEEE Trans. Syst., Man, Cybern. A, Syst. Humans, vol. 28, no. 5, pp. 562-574, Sep. 1998.

[37] D. Wilkie, J. van den Berg, and D. Manocha, "Generalized velocity obstacles," in Proc. IEEE/RSJ Int. Conf. Intell. Robots Syst., Oct. 2009, pp. 5573-5578.

[38] J. Kiusalaas, Numerical Methods in Engineering With MATLAB. Cambridge, U.K.: Cambridge Univ. Press, 2007.

[39] C. Poussot-Vassal, O. Sename, L. Dugard, and S. M. Savaresi, "Vehicle dynamic stability improvements through gain-scheduled steering and braking control," Vehicle Syst. Dyn., vol. 49, no. 10, pp. 1597-1621, Oct. 2011

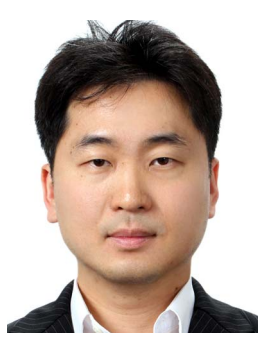

Suhyeon Gim received the Ph.D. degree in intelligent robotic vehicles in 2017. He has accumulated experience on the mechanism and control for the robotic hardware as well as software for dealing with various kinds of robotics platform. He has worked as a Researcher with the ISRI/SKKU and the Institut Pascal, University Clermont Auvergne on the algorithmic development of autonomous navigation vehicle with safe and human-like path planning. $\mathrm{He}$ is working as a Principal Research Engineer for robotics and automation in South Korea.

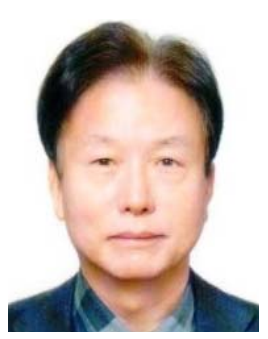

Sukhan Lee (Life Fellow, IEEE) received the B.S. and M.S. degrees in electrical engineering from Seoul National University, South Korea, in 1972 and 1974, respectively, and the Ph.D. degree in electrical engineering from Purdue University, West Lafayette, USA, in 1982. From 1983 to 1997 , he was with the Department of Electrical Engineering and Computer Science, University of Southern California, as a Professor, and from 1990 to 1997, he was with the Jet Propulsion Laboratory/NASA, California Institute of Technology, as Senior MTS. From 1998 to 2003, he was the Executive Vice President and the Chief Research Officer with the Samsung Advanced Institute of Technology. Since 2003, he has been a Professor of information and communication engineering as well as of artificial intelligence with Sungkyunkwan University. His research interest is in the areas of cognitive robotics, intelligent systems, deep learning, and micro/nano electro-mechanical systems. He is currently a Life Fellow of Korea National Academy of Science and Technology.

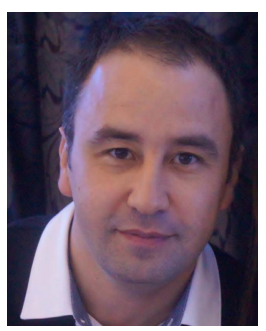

Lounis Adouane (Member, IEEE) received the $\mathrm{Ph} . \mathrm{D}$. degree in automatic control from the FEMTO-ST Laboratory, ENSMM, France, in 2005, and the H.D.R. (habilitation to steer research in robotics) degree from Blaise Pascal University, in 2015. During his Ph.D. studies, he deeply investigated the field of mobile multi robot systems, especially those related to bottom-up and decentralized control architectures. In 2005, he joined the Ampere Laboratory, INSA Lyon, and he studied hybrid (continuous/discrete) control architectures applied to cooperative mobile robots arms. From 2006 to 2019, he was an Associate Professor with the Institut Pascal, Polytech Clermont-Ferrand. He is currently a Full Professor with the Heudiasyc, UMR 7253 CNRS/Universite de Technologie de Compiegne. From 2018 to 2019, he was the Head of ISPR Group (Images, Perception Systems and Robotics, 80 members), Institut Pascal. Since 2018, he is a member of the Technical Committee of IFAC-Intelligent Autonomous Vehicles and serves in the Editorial Board Member of Journal of Intelligent and Robotic Systems. He had the opportunity to visit during few months several institutions/laboratories, such as in 2009 LIST (Luxembourg), in 2014 Cranfield and Kingston universities (U.K), and in 2018 KIT (Germany). He is the author/coauthor of more than 100 refereed international articles and two books. His main research interests include planning and control of mobile robots/intelligent vehicles (autonomous and clean), decision-making under uncertainty, hybrid (continuous/discrete) and hybrid (reactive/cognitive) multi controller architectures, Lyapunov-based synthesis and stability, cooperative multi robot systems, artificial intelligence (e.g., MDP or MAS approaches), and energy management (e.g., optimal control or neuro-fuzzy approaches). 